\title{
Etude de la flexion des coques toriques d'épaisseur constante chargées axisymétriquement
}

\author{
Application au calcul des soufflets toriques
}

\section{A study of the bending of constant thickness toroidal shells under axi-symmetrical loading}

Application to toroidal bellows joint design

PAR

S. X. CASACCI

\author{
INGÉNIEUR-DOCTEUR
}

CHEF DU DÉPARTEMENT TURRINES DES ÉTABI.ISSEMENTS NEYHPIC:
ET

L'étude unalytique de la flexion axisymétrique' des coques toriques demeare très complexe. mème si l'on se limite aur petites déformations élastiques.

Des solutions analyfiques upprnchees peuvenl être obtenues, pour certains cas de charge. sous forme d'une combinaison linéaire de denx fonctions de Hankel d'ordre 1/3. Pour les autres cas de charge, la solution particulière s'exprime soit à l'aide d'une série de Fourier dont les coefficients se cafculent à l'aide de fractions continues (V. Y. Novozhilov), soit à l'aide d'une combinaison de fonctions simples et d'une fonction de Lommel (R.A. Clarh).

La méthode que nous exposons consiste à transformer les équations de base en un système d'équations intégrales de Fredholm. Des solutions analytiques de ce système peuvent ètro obtenues à l'aide d'expressions asymptotiques. Nouss n'examinerons cependant dans cette étude qu'une résolution numérique appliquée al calcul des contraintes et des déformations d'un élément de sonfflet torique soumis à des charges rxiules.

\begin{abstract}
The analytical study of the axi-symmetrical bending of toroidal shells is always highly comple $x$, even if only small elastic strains ar" considered.

For certain loading cases, approximate analylical solutions can be found in the form of " linear combination of two Hankel functions of order 1/3. The particular solutions for other loading cases are either expressed by means of a Fourier series, the coefficients of which are calculated by means of continuous fraclions (V.V. Novozhilov), or by combining simple functions and a Lommel function (R. A. Clark).

In the method described, the basic equations are transformed into a Fredholn integral equalion system, analyfical solutions of which can be obtained with the aid of asymptotic expressions. The anthors restrict themselves to an examination of a numerical solution, which is applied to the calculation of stresses and strains in a toroidal bellows element undergoing axial lording.
\end{abstract}

\section{INTRODUCTION}

Les coques loriques sont très utilisées dans la construction mécanique et la grosse chaudronnerie. Citons par exemple:

- Volutes de pompes et de turbo-compresseurs;

- Bâches de turbines hydrauliques, à vapeur ou à gaz;

- Raccordement des coques de révolution entre elles;
- Soufflets métalliques;

-- Joints de conduites type Oméga, etc.

L'étude théorique de la flexion des coques toriques est d'une grande complexité. Les premiers travaux de $H$. Wissler [12] se rapportent à des cas de charge particuliers. La méthode de résolution de cet auteur, basée sur des développements en série, nécessite de très longs calculs. R. A. Clark [4] traite les mêmes cas de charge 
que $H$. Wissler pour vérifier la précision de sa méthode d'intégration asymptotique. Il applique ensuite ce procédé de résolution au calcul des soufllets métalliques et des joints Omćga. Cette méthode ne s'applique qu'aux coques toriques dont le rayon d'enroulement (rayon moyen) est grand par rapport au rayon de la section méridienne

$$
\left(\lambda=\frac{b}{a} \text { grand }\right)
$$

La méthode que nous exposons dans cette ćtude consiste à transformer les équations de base en un système d'équations intégrales de Fredholm et à résoudre numériquement ce système [1]. Nous appliquons cette méthode au calcul des contraintes et des déformations pour les éléments de tores qui peurent constituer un soufflet torique. Nous donnons des résultats pour des charges axiales équilibrées; cependant la méthode s'applique à tous les cas de charge.

\section{I. - NOTATIONS GENÉRALES (voir fig. 1)}

$r_{1}$ : premier rayon principal de courbure en $\mathrm{P}$; rayon du cercle méridien du tore $r_{1}=a$;

$r_{2}:$ deuxic̀me rayon principal de courbure en $\mathrm{P}$

$$
r_{\ddot{\prime}}=\frac{\lambda+\sin \vartheta}{\sin \varphi} a
$$

$b$ : rayon moyen du tore;

$\lambda: \frac{b}{a}$;

$r_{0}$ : vayon d'un parallèle

$$
r_{0}=b+a \sin \varphi=a(\lambda+\sin \varphi) ;
$$

$\%$ : angle que fait la normale en $P$ avec l'axe de révolution;

$\theta$ : longitude de $P$ par rapport au méridien origine;

$e$ : épaisseur de la coque;

$\nu$ : coefficient de Poisson;

E : module d’élasticité du matériau;

$\mathrm{D})$ : rigidité de flexion $\mathrm{D}=\frac{\mathrm{E} e^{3}}{12\left(1-v^{2}\right)}$

$\mathrm{D}_{x}$ : rigidité de traction $\mathrm{D}_{X}=\frac{\mathrm{E} e}{1-\mathrm{v}^{2}}$

$\mathrm{N}$ : effort normal agissant sur un élément unité de parallèle;

$n$ : effort normal agissant sur un élément unité de méridien;

Q : effort de cisaillement agissant sur un élément unité de parallèje;
M : moment de llexion agissant sur un écément unité de parallèle;

$m$ : moment de flexion agissant sur un élément unité de méridien;

$x=r_{2} Q=\frac{r_{0} Q}{\sin \varphi}$ effort de cisaillement par radiatn;

p : pression posilive si elle a mème sens que la normale extérieure au point d'applicalion ;

$s_{\varphi}$ : allongement relatif en un point du méridien de la surface moyenne;

$\varepsilon_{\theta}$ : allongement relatif d'un parallele de la surface moyenme;

९.: variation de courbure du méridien de la surface moyenne;

$\rho_{\theta}$ : variation de courbure du parallèle de la surface moyenne;

v : déplacement tangentiel, posilif dans le sens des croissants;

w) : déplacement normal, positif s'il a mème sens que la normale intérieure;

$y$ : déformation angulaire, angle des tangentes au méridien avant et après déformalion;

$k=12\left(1-\mathrm{y}^{2}\right)\left(\frac{a}{h}\right)^{2}=\frac{\mathrm{Eea}^{2}}{\mathrm{D}}$

Les variables efforts et déformations affectes des indices 1 ou 2 se rapportent aux parallèles définis par $\varphi=\varphi_{1}$ ou $\varphi=\varphi_{2}$. 


\section{II. - EQUATIONS GENERALES DES COQUES TORIQUES}

\section{II.1. Equations d'équilibre.}

L'équilibre d'un anneau élémentaire limité par (fig. 1) deux parallèles infiniment voisins $९$ et $\varphi+d \varphi$ donne la relation

$\frac{d}{d \varphi} r_{0}(\mathrm{~N} \sin \varphi+\mathrm{Q} \cos \varphi)=p a r_{0} \cos \varphi$

et l'équilibre d'un demi-anneau (fig. 2 et 3 ) les relations :

$\frac{d}{d \varphi} \cdot r_{0}(\mathrm{Q} \sin \varphi-\mathrm{N} \cos \varphi)=$ par $_{0} \sin \varphi-n a$

$\frac{d}{d \varphi}\left(r_{0} \mathrm{M}\right)-m a \cos \varphi-\mathrm{Q} a r_{0}=0$

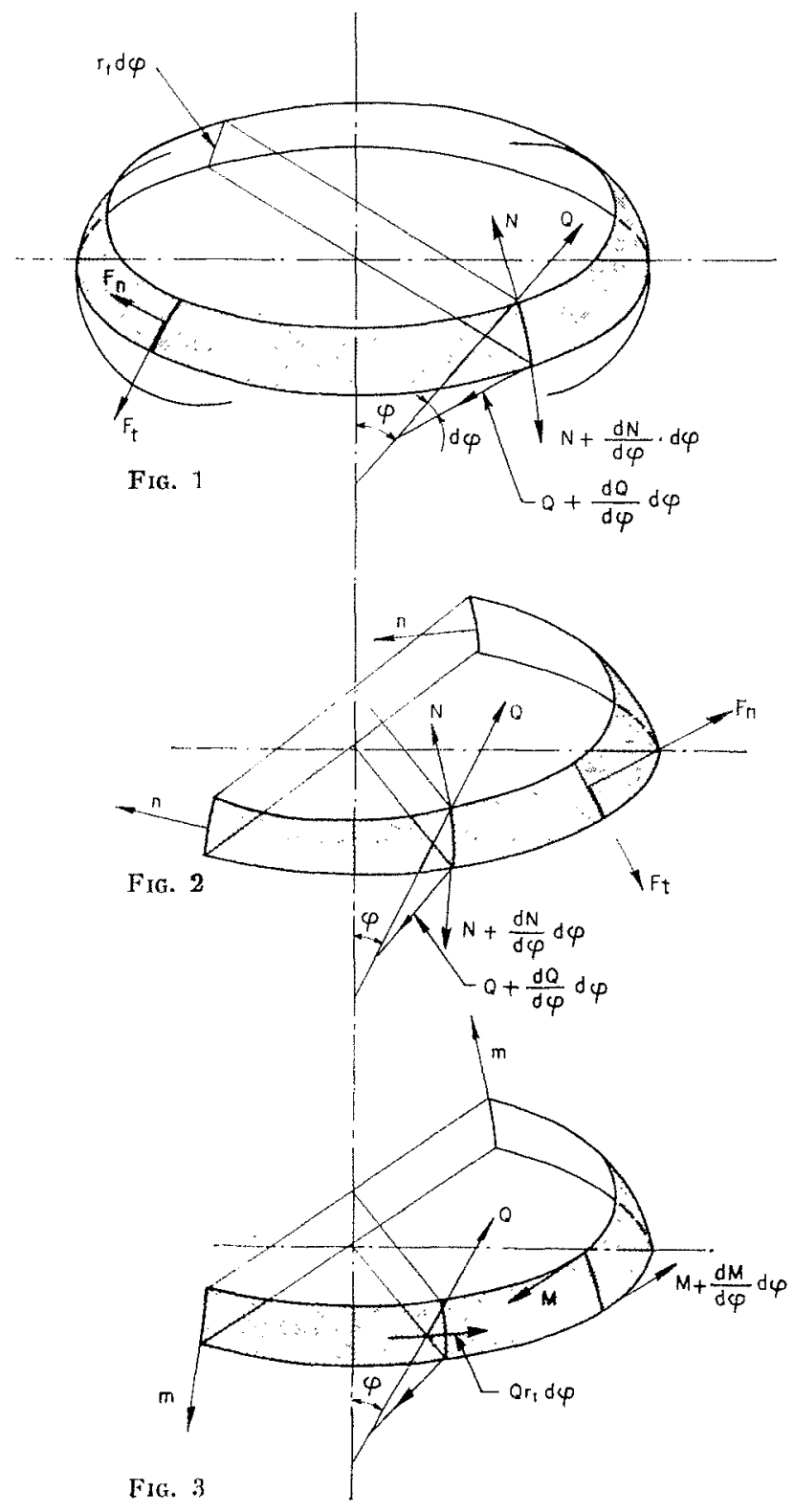

II.2. Expression des déformations (fig. 4 et 5). Allongement unitaire du méridien :

$$
\varepsilon_{\varphi}=\frac{1}{a}\left(\frac{d Y}{d \varphi}-w\right)
$$

Allongement unitaire d'un parallèle :

$$
\varepsilon_{\theta}=\frac{1}{r_{0}}(v \cos \varphi-w \sin \varphi)
$$

ou encore :

$$
\varepsilon_{\theta}=\frac{1}{r_{2}}(v \operatorname{cotg} \varphi-w)
$$


Rotation de la tangente à la méridienne :

$$
y=\frac{1}{a} \frac{d w}{d \varphi}+\frac{v}{a}
$$

Changement de courbure le long d'un méridien :

$$
\rho_{\varphi}=\frac{1}{a} \frac{d}{d \varphi}\left(\frac{d w}{a d \varphi}+\frac{v}{a}\right)=\frac{1}{a} \frac{d y}{d \varphi}
$$

Variation de courbure d'un parallèle due à la flexion :

$$
i_{\theta}=\frac{y \operatorname{cotg} \varphi}{r_{2}}
$$

\section{II.3. Relations entre les efforts et les défor- mations.}

Les relations exprimant la loi de Hooke donnent :

$$
\begin{aligned}
\varepsilon_{\theta} & =\frac{1}{\mathrm{E} e}(\mathrm{~N}-\mathrm{v} n) \\
\varepsilon_{\theta} & =\frac{1}{\mathrm{~F} e}(n-\mathrm{vN})
\end{aligned}
$$

et inversement :

$$
\begin{aligned}
& \mathrm{N}=\frac{\mathrm{E} e}{1-\nu^{2}}\left(\varepsilon_{\varphi}+\nu \varepsilon_{\theta}\right)=\mathrm{D}_{\mathrm{X}}\left(\varepsilon_{\varphi}+\nu \varepsilon_{\theta}\right) \\
& n=\frac{\mathrm{E} e}{1-\nu^{2}}\left(\varepsilon_{\theta}+\nu \varepsilon_{\varphi}\right)=\mathrm{D}_{\mathrm{N}}\left(\varepsilon_{\theta}+\nu \varepsilon_{\varphi}\right) \\
& \vartheta_{\varphi}=\frac{1}{\mathrm{D}}(\mathrm{M}-\nu m) \\
& \rho_{\theta}=\frac{1}{\mathrm{D}}(m-\nu \mathrm{M})
\end{aligned}
$$

et inversement :

$$
\begin{aligned}
& \mathrm{M}=-\mathrm{D}\left(\rho_{\varphi}+v_{\varphi_{\theta}}\right) \\
& m=-\mathrm{D}\left(\varphi_{\theta}+v_{\varphi}\right)
\end{aligned}
$$

soit, en tenant compte des relations de courbure (2-8) et (2-9),

$$
\begin{aligned}
& \mathrm{M}=-\frac{\mathrm{D}}{a}\left(\frac{d y}{d \varphi}+\nu \frac{\cos \varphi}{\lambda+\sin \varphi} y\right) \\
& m=-\frac{\mathrm{D}}{a}\left(\nu \frac{d y}{d \varphi}+\frac{\cos \varphi}{\lambda+\sin \varphi} y\right)
\end{aligned}
$$

\section{II.4. Equations différentielles de base.}

L'équation d'équilibre des moments (2-3) s'écrit, compte tenu des relations (2-18) et (2-19)

$$
\begin{aligned}
\frac{d}{d \varphi}\left[(\lambda+\sin \varphi) \frac{d y}{d \varphi}\right] & -\frac{\lambda \sin \varphi+1}{\lambda+\sin \varphi} y \\
& +(1-\nu) y \sin \varphi=-\frac{a x}{\mathrm{D}} \sin \varphi
\end{aligned}
$$

Les équations (2-6) et (2-11) permettent d'écrire :

$$
(n-\nu \mathrm{N}) \frac{r_{0}}{\sin \varphi}=\mathrm{E} e(v \operatorname{cotg} \varphi-w)
$$

soit en dérivant par rapport à o

$$
\begin{aligned}
& \frac{d}{d \varphi}\left[\frac{r_{0}}{\sin \varphi}\left(n-\nu_{\mathrm{N}}\right)\right] \\
&=\mathrm{E} e\left[\operatorname{cotg} \varphi \frac{d v}{d \varphi}-\frac{v}{\sin ^{2} \varphi}-\frac{d w^{-}}{d \varphi}\right]
\end{aligned}
$$

L'élimination de $w$ entre les relations $(2-5)$ et (2-6) donne :

$$
\begin{aligned}
& a\left(\mathrm{~N}-y_{n}\right) \operatorname{cotg} \varphi-r_{0}(n-\nu \mathrm{N}) \frac{\cos \varphi}{\sin ^{2} \varphi} \\
& =\mathrm{E} e\left(\frac{d v}{d \varphi} \operatorname{cotg} \varphi-v \operatorname{cotg}^{2} \varphi\right)
\end{aligned}
$$

En retranchant $(2-22)$ de $(2-23)$ on obtient finaJement :

$$
\begin{aligned}
a(\mathrm{~N}-\nu n) \operatorname{cotg} \varphi-\frac{1}{\sin \varphi} \frac{d}{d \varphi}\left[r_{0}(n-v \mathrm{~N})\right] & =\mathrm{E} e a !
\end{aligned}
$$

L'équation d'équilibre (2-1), intégréc par rapport à $\varphi$ devient :

$$
r_{0} \mathrm{~N} \sin \varphi+r_{0} \mathrm{Q} \cos \varphi=\mathrm{A}+\mathrm{S}
$$

avec :

$\mathrm{S}=a \int p r_{0} \cos \varphi d \varphi$

$A=$ constante d'intégration dépendant des conditions aux limites.

L'expression (2-25) s'écrit encore :

$$
N=\frac{A+S}{r_{0} \sin \varphi}-\frac{x \cos \varphi}{r_{0}}
$$

où

$$
x=\frac{r_{0} Q}{\sin \varphi}
$$

En portant $\mathrm{N}$ dans l'équation (2-2), on obtient :

$$
n=-\frac{1}{a} \frac{d x}{d \varphi}-\frac{\mathrm{A}+\mathrm{S}}{a \sin ^{2} \varphi}+\frac{p r_{0}}{\sin \varphi}
$$


ou encore :

Si nous posons :

$$
n=-\frac{1}{a} \frac{d x}{d \varphi}-\frac{\mathrm{A}}{a \sin ^{2} \varphi}+\frac{p a}{2}
$$

L'équation (2-24) se transforme en :

$$
\begin{aligned}
& \frac{d}{d \varphi}\left[(\lambda+\sin \varphi) \frac{d x}{d \varphi}\right]-\frac{\lambda \sin \varphi+1}{\lambda+\sin \varphi} x+(1+\nu) x \sin \varphi \\
&=\text { Ee ayj } \sin \varphi+A \lambda \frac{\cos \varphi}{\sin ^{3} \varphi} \\
&\left(2+\frac{\sin \varphi}{\lambda+\sin \varphi}\right)-\frac{p a^{2}}{2} \lambda \frac{\cos \varphi}{\lambda+\sin \varphi}
\end{aligned}
$$

Les équations (2-20) et (2-29) constituent les équations différentielles de base.
$\mathrm{L}()=.\frac{d}{d \varphi}\left[(\lambda+\sin \varphi) \frac{d(.)}{d \varphi}\right]-\frac{\lambda \sin \varphi+1}{\lambda+\sin \varphi}($.

ces équations prennent la forme :

$$
\mathrm{L}(y)+(1-\mathrm{v}) y \sin \varphi=-\frac{a x}{\mathrm{D}} \sin \varphi(2-31)
$$

$\mathrm{L}(x)+(1+\nu) x \sin \varphi=\mathrm{E} e$ ay $\sin \varphi+\mathrm{A} \frac{\cos \varphi}{\sin ^{3} \varphi}$

$$
X\left(2+\frac{\sin \varphi}{\lambda+\sin \varphi}\right) \lambda-\frac{p a^{2}}{2} \frac{\lambda \cos \varphi}{\lambda+\sin \varphi}
$$

\section{III. - TRANSFORMATION DES EQUATIONS DE BASE EN UN SYSTËME DE DEUX EQQUATIONS INTÉGRALES DE FREDHOLM}

\section{III.1. Définition des variables.}

Nous poserons :

$\mathrm{T}=\frac{x}{\mathrm{~F}}$

$V=\frac{\mathrm{D}}{a \mathrm{~F}} y$

$\mathrm{T}$ et $\mathrm{V}$ sont des variables sans dimension;

$F$, effort de référence, défini pour chaque cas de charge étudié;

$F$ est égal par exemple à $M_{1}, M_{2}, r_{0} X_{1}$, etc.

Les équations (2-31) et (2-32) deviennent :

$\mathrm{L}(\mathrm{V})+(1-v) \mathrm{V} \sin \varphi=-\mathrm{T} \sin \varphi$

$\mathrm{L}(\mathrm{T})+(1+v) \mathrm{T} \sin \varphi=k \mathrm{~V} \sin \varphi+\mathrm{F}(\varphi, p)$

avec :

$$
\begin{aligned}
\mathrm{F}(\varphi, p) & =\frac{\mathrm{A}}{\mathrm{F}} \lambda \frac{\cos \varphi}{\sin ^{3} \varphi}\left(2+\frac{\sin \varphi}{\lambda+\sin \varphi}\right) \\
& -\frac{p a^{2}}{2 \mathrm{~F}} \frac{\lambda \cos \varphi}{\lambda+\sin \varphi}
\end{aligned}
$$

\section{III.2. Conditions aux limites.}

Les conditions aux limites relatives à $r$ se ramènent à :

$$
\begin{array}{lll}
q=0 & \mathrm{~T}=\mathrm{T}_{1} & \text { en } \vartheta_{1} \\
q=0 & \mathrm{~T}=\mathrm{T}_{2} & \text { en } \vartheta_{2}
\end{array}
$$

par le changement de variable :

$$
\mathrm{T}=q+f(\varphi)
$$

Les conditions aux limites relatives à $V$ se transforment en :

$$
\left.\begin{array}{lll}
u=0 & y=y_{1} & \text { en } p_{1} \\
u=0 & y=y_{2} & \text { en } \varphi_{2}
\end{array}\right\}
$$

ou plus généralement en :

$$
\begin{array}{ll}
u^{\prime}+v \frac{\cos \varphi}{\lambda+\sin \varphi} u=0 & \mathrm{M}=\mathrm{M}_{1} \quad \text { en } \varphi_{1} \\
u^{\prime}+v \frac{\cos \varphi}{\lambda+\sin \varphi} u=0 & \mathrm{M}=\mathrm{M}_{2}
\end{array}
$$

par le changement de variable :

$$
\mathrm{V}=u+g(\varphi)
$$


Le choix des fonctions $f(\varphi)$ et $g(\varphi)$ est tel que :

$$
\mathrm{L}(f)=\mathrm{L}(g)=0
$$

Les solutions linéairement indépendantes de l'équation différentielle

$\mathrm{L}(\Omega)=\frac{d}{d \varphi}\left[(\lambda+\sin \varphi) \frac{\mathrm{D} \Omega}{d \varphi}\right]-\frac{\lambda \sin \varphi+1}{\lambda+\sin \varphi} \Omega=0$

sont de la forme :

$$
\psi=\frac{1}{\lambda+\sin \varphi}
$$

et

$$
\zeta=\psi \int \frac{e^{-\int \frac{\cos \varphi}{\lambda+\sin \varphi}} d \varphi}{\psi^{2}} d \varphi=\frac{\lambda \varphi-\cos \varphi}{\lambda+\sin \varphi}
$$

Les fonctions $f$ et $g$ pourront alors s'exprimer par :

$$
\begin{aligned}
& f(\varphi)=\frac{A_{1}}{\lambda+\sin \varphi}+A_{2} \frac{\lambda \varphi-\cos \varphi}{\lambda+\sin \varphi} \\
& g(\varphi)=\frac{B_{1}}{\lambda+\sin \varphi}+B_{2} \frac{\lambda \varphi-\cos \varphi}{\lambda+\sin \varphi}
\end{aligned}
$$

où les constantes $A_{1}, A_{2}, B_{1}$ et $B_{2}$ dépendent des conditions limites.

\section{III.3 Equations intégrales.}

Avec les changements de variables (3-7) et (3-10), les équations (3-3) et 3-4) deviennent :

$\mathrm{L}(u)=-(1-y) \mathrm{V} \sin \varphi-\mathrm{T} \sin \varphi$

$\mathrm{I}(q)=-(1+\nu) \mathrm{T} \sin \varphi+k \mathrm{~V} \sin \varphi+\mathrm{F}(\varphi, p)$

Soit, sous forme d'équations intégrales :

$$
\begin{aligned}
& u(\varphi)=(1-\nu) \int_{\varphi_{1}}^{\varphi_{y}} \mathrm{~K}_{2}(\varphi, \alpha) \mathrm{V}(\alpha) \sin \alpha d \alpha+\int_{\varphi_{1}}^{\varphi_{\mu}} \mathrm{K}_{2}(\varphi, \alpha) \mathrm{T}(\alpha) \sin \alpha d \alpha \\
& \eta(\varphi)=(1+\nu) \int_{\varphi_{1}}^{\varphi_{2}} \mathrm{~K}_{1}(\varphi, \alpha) \mathrm{T}(\alpha) \sin \alpha d \alpha-k \int_{\varphi_{1}}^{\varphi_{\alpha}} \mathrm{K}_{1}(\varphi, \alpha) \mathrm{V}(\alpha) \sin \alpha d \alpha-\int_{\varphi_{1}}^{\varphi_{3}} \mathrm{~K}_{1}(\varphi, \alpha) \mathbf{F}(\alpha, p) d \alpha
\end{aligned}
$$

ou encore en posant :

$$
\begin{gathered}
\int_{\varphi_{1}}^{\varphi_{2}} \mathrm{~K}_{1}(\varphi, \alpha) \mathrm{F}(\alpha, p) d \alpha=\Phi(p, \varphi) \\
\mathrm{V}(\varphi)=\int_{\varphi_{1}}^{\varphi \alpha} \mathrm{K}_{2}(\varphi, \alpha)[(1-\nu) \mathrm{V}(\alpha) \sin \alpha+\mathrm{T}(\alpha) \sin \alpha] d \alpha+g(\varphi) \\
\mathrm{T}(\varphi)=\int_{\varphi_{1}}^{\varphi_{\alpha}} \mathrm{K}_{1}(\varphi, \alpha)[(1+\nu) \mathrm{T}(\alpha) \sin \alpha-k \mathrm{~V}(\alpha) \sin \alpha] d \alpha+f(\varphi)-\Phi(p, \varphi)
\end{gathered}
$$

$\mathrm{K}_{1}(\varphi, \alpha)$ et $\mathrm{K}_{2}(\varphi, \alpha)$ sont les noyaux relatifs à $\mathrm{V}$ et $\dot{\mathrm{a}} \mathrm{T}$.

\section{III.4. Détermination des noyaux.}

Les opérateurs différentiels des équations $(3-15)$ et (3-16) sont de la forme :

$$
\mathrm{L}(\Omega)=\frac{d}{d \varphi}\left[p(\varphi) \frac{d \Omega}{d \varphi}\right]+h(\varphi) . \Omega
$$

Si $\Omega_{1}(\varphi)$ et $\Omega_{2}(\varphi)$ sont deux solutions particu- lières de $L(\Omega)=0$, l'une satisfaisant à la condition limite en $\varphi_{1}$ et l'autre à la condition en $\varphi_{9}$, la fonction d'influence correspondante se note:

$$
G(\varphi, \alpha)= \begin{cases}\frac{\Omega_{1}(\varphi) \cdot \Omega_{2}(\alpha)}{c} & \varphi_{1} \leqslant \varphi \leqslant \alpha \\ \frac{\Omega_{2}(\varphi) \cdot \Omega_{1}(\alpha)}{c} & x \leqslant \varphi \leqslant \varphi_{2}\end{cases}
$$


où $c$ désigne une constante définie par :

$$
c=-p(0)\left[\Omega_{1}(\varphi) \Omega^{\prime}{ }_{2}(\varphi)-\Omega_{2}(\varphi) \Omega^{\prime}{ }_{1}(\varphi)\right]
$$

G, conme fonction de $\breve{y}$, satisfait aux conditions limites.

Rappelons que :

$L(\Omega)=\frac{d}{d \varphi}\left[(\lambda+\sin \varphi) \frac{d \Omega}{d \varphi}\right]-\frac{\lambda \sin \varphi+1}{\lambda+\sin \vartheta} . \Omega$ admet deux solutions particulières de la forme

$$
\Omega=\frac{C}{\lambda+\sin \varphi}+C \frac{\lambda-\cos \varphi}{\lambda+\sin \varphi}
$$

\section{III.4.1. NOYAU RELATIF A T.}

Les conditions de cisaillement nul aux limites imposent :

$$
\begin{aligned}
& \Omega_{1}(\varphi)=0 \quad \text { en } \varphi_{p}=\varphi_{1} \\
& Q_{2}(\varphi)=0 \quad \text { en } p=p_{p}
\end{aligned}
$$

D’une façon générale $\Omega=0$ donne :

$$
\frac{\mathrm{C}^{\prime}}{\mathrm{C}}=-\frac{1}{2 \varphi-\cos \varphi}=?
$$

Soit pour

$$
\frac{\mathrm{C}_{1}^{\prime}}{\mathrm{C}_{1}}=-\frac{1}{\lambda_{\varphi_{1}}-\cos \varphi_{1}}=\varphi_{1}
$$

et pour $\varphi=\varphi_{2}$

$$
\frac{C_{2}^{\prime}}{C_{2}}=-\frac{1}{2 p_{2}-\cos \varphi_{2}}=?_{2}
$$

D'où :

$$
\Omega_{1}(\varphi)=\frac{C_{1}}{\lambda+\sin \varphi}\left[1+\rho_{1}(\lambda \varphi-\cos \varphi)\right]
$$

$$
Q_{2}(\varphi)=\frac{C_{2}}{\lambda+\sin \varphi}\left[1+\rho_{2}(\lambda \varphi-\cos \varphi)\right]
$$

On en déduit la valeur de la constante $c$ donnée par $(3-23)$.

$$
c=\mathrm{C}_{1} \mathrm{C}_{2}\left(p_{1}-\cdots+q_{2}\right)
$$

D'où le premier noyau :

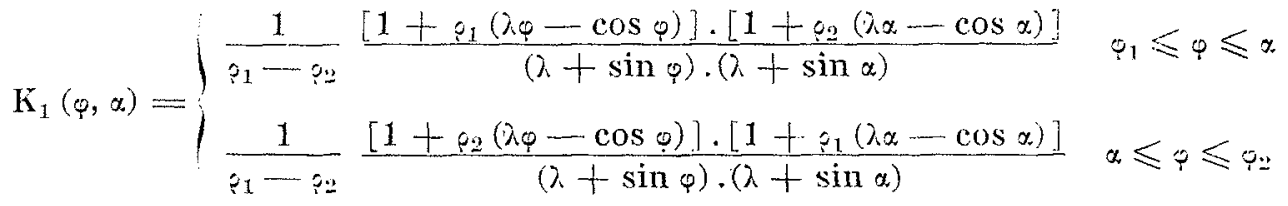

\section{III.4.2. Noyau RELATIF A $Y$.}

Les conditions de moment nul aux limites imposent :

$$
\left.\begin{array}{ll}
\Omega_{1}^{\prime}+v \frac{\cos \varphi}{\lambda+\sin \varphi} \Omega_{1}=0 & \text { en } \varphi=\varphi_{1} \\
\Omega_{2}^{\prime}+\nu \frac{\cos \varphi}{\lambda+\sin \varphi} \Omega_{2}=0 & \text { en } \varphi=\varphi_{2}
\end{array}\right\}
$$

Des solutions de la forme :

$$
\Omega=\frac{D}{\lambda+\sin \varphi}+D^{\prime} \frac{\lambda \varphi-\cos \varphi}{\lambda+\sin \varphi}
$$

donnent d'une facon générale :

$$
\frac{D^{\prime}}{D}=\frac{1}{\left[(\lambda+\sin \varphi)^{2} /(1-\nu) \cos \varphi\right]-(\lambda \varphi-\cos \varphi)}=\mu .
$$

Soit, pour $\varphi=\varphi_{1}$,

$$
\frac{\mathrm{D}_{1}^{\prime}}{\mathrm{D}_{1}}=\mu_{1}=\frac{(1-\nu) \cos \varphi_{1}}{\left(\lambda+\sin \varphi_{1}\right)^{2}-(1-\nu)\left(\lambda_{1}-\cos \varphi_{1}\right) \cos \varphi_{1}}
$$

et pour $\varphi=\varphi_{2}$

$$
\frac{\mathrm{D}_{2}^{\prime}}{\mathrm{D}_{2}}=\mu_{2}=\frac{(1-y) \cos \varphi_{2}}{\left(\lambda+\sin \varphi_{2}\right)^{2}-(1-\nu)\left(\lambda_{\varphi_{2}}-\cos \varphi_{2}\right) \cos \varphi_{2}}
$$


Comme au paragraphe précédent, on en déduit la valeur de la constante $c$

$$
c=\mathrm{D}_{1} \mathrm{D}_{2}\left(\mu_{1}-\psi_{2}\right)
$$

et du deuxième noyau :

$$
\mathrm{K}_{2}(\varphi, x)=\left\{\begin{array}{l}
\frac{1}{\mu_{1}-\mu_{1}} \frac{\left[1+\mu_{1}(\lambda \varphi-\cos \varphi)\right] \cdot\left[1+\mu_{2}(\lambda \alpha-\cos \alpha)\right]}{(\lambda+\sin \varphi) \cdot(\lambda+\sin x)} \varphi_{1} \leqslant 0 \leqslant x . \\
\frac{1}{\mu_{1}-\mu_{2}} \frac{\left[1+\mu_{2}(\lambda \varphi-\cos \varphi)\right] \cdot\left[1+\mu_{1}(\lambda \alpha-\cos \alpha)\right]}{(\lambda+\sin \varphi) \cdot(\lambda+\sin x)} x \leqslant 0 \leqslant \varphi_{2}
\end{array}\right.
$$

Remarque. - Dans le cas où les conditions limites relatives à $V$ sont de la forme $V=0$ en $\varphi_{1}$ ou en $\varphi_{2}$ (rotation nulle) les constantes $\mu_{1}$ ou $\mu_{2}$ se transforment respectivement en $i_{1}$ ou $\varphi_{2}$.

\section{III.5. Cas de charges étudiés (fig. 6).}

Les cas de charges pratiques peuvent se décomposer en cas types simples :

Nous considérerons les cas types suivants :

Cas 1. - Effort radial appliqué à la base $1\left(\varphi_{\varphi}=\varphi_{1}\right)$;

Cas 2. -.. Effort radial appliqué à la base $2\left(\%=\varphi_{2}\right)$;

Cas 3. - Efforts axiaux équilibrés appliqués aux deux bases;

Cas 4. -... Pression uniforme équilibrée par un effort axial sur la base 1;

Cas 5. -- Moment agissant sur la base 1;

Cas 6. - Moment agissant sur la base 2.
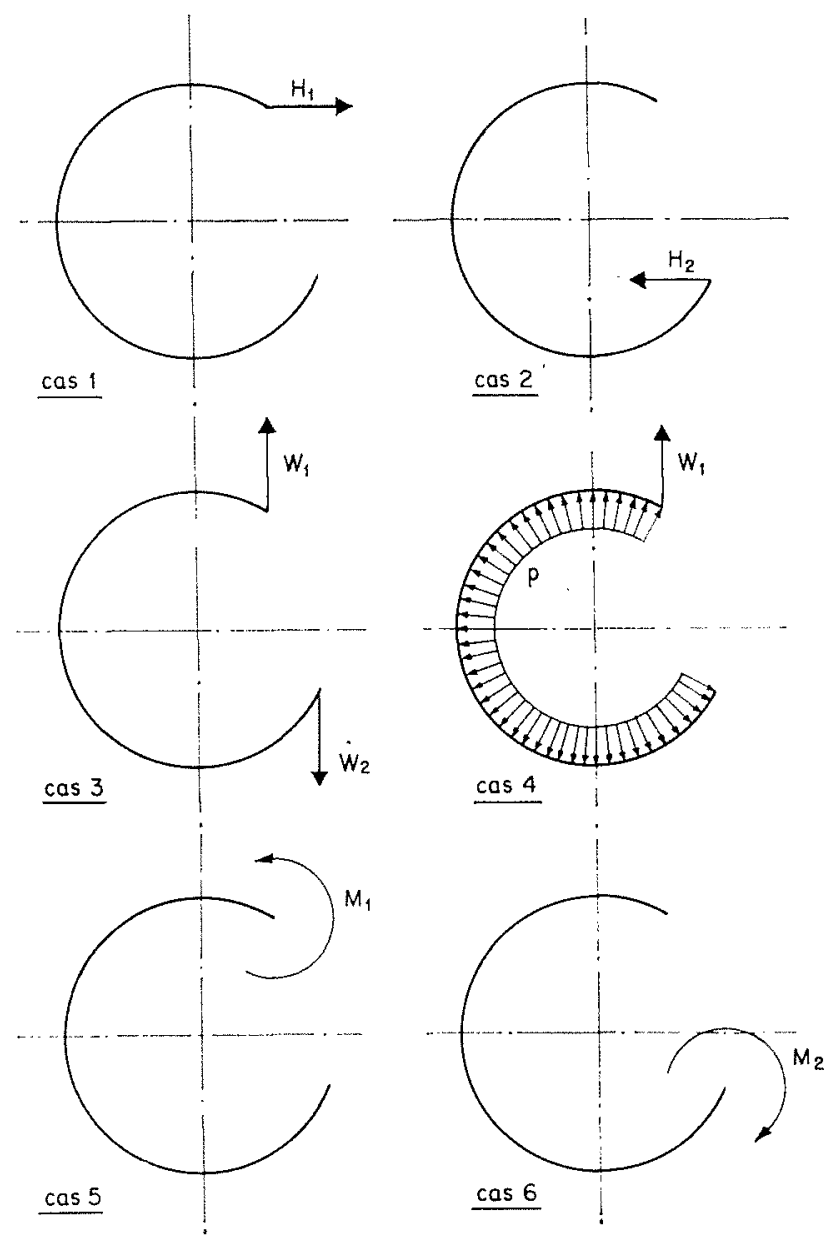

Fug, 6 


\section{III.6. Détermination générale des seconds membres des équations intégrales.}

Les seconds membres des équations intégrales (3-20) et (3-21) sont constitués respectivement par les fonctions $g(\varphi)$ et $f(\varphi)-\Phi(p, \varphi)$.

Nous calculerons ces fonctions dans le cas général pour les conditions limites suivantes :

$$
\begin{array}{lll}
\mathrm{T}=\mathrm{T}_{1} & & \text { en } \varphi=\varphi_{1} \\
\mathrm{~T}=\mathrm{T}_{2} & & \text { en } \varphi=\varphi_{2} \\
\mathrm{M}=\mathrm{M}_{1} & \text { ou } \mathrm{V}=0 & \text { en } \varphi=\varphi_{1} \\
\mathrm{M}=\mathrm{M}_{2} & \text { ou } \mathrm{V}=0 & \text { en } \varphi=\varphi_{2}
\end{array}
$$

Nous déterminerons ensuite les valeurs de $T_{1}, T_{2}, M_{1}$ et $M_{2}$ correspondant aux cas de charges envisagés au chapitre III-5.

III.6.1. Calcul de $\Phi(p, 0)$.

On sait que :

$$
\Phi(p, \varphi)=\int_{\varphi_{1}}^{\varphi_{2}} \mathrm{~K}_{1}(\vartheta, x) \mathrm{F}(p, \alpha) d \alpha
$$

avec :

$$
\mathrm{F}(p, \alpha)=\frac{\mathrm{A}}{\mathrm{F}} \lambda \frac{\cos \alpha}{\sin ^{3} \alpha}\left(2+\frac{\sin \alpha}{\lambda+\sin \alpha}\right)-\frac{p a^{2}}{2} \frac{\lambda \cos \alpha}{\lambda+\sin \alpha}
$$

et $\mathrm{K}_{1}(\varphi, \alpha)$ donné par (3-32).

Tous calculs faits, on obtient :

$$
\Phi(p, \varphi)=\frac{\lambda}{\left(\rho_{1}-\rho_{2}\right)(\lambda+\sin \varphi)}\left\{\begin{array}{l}
-\left(\rho_{1}-\rho_{2}\right)\left[\frac{\mathrm{A}}{\mathrm{F}} \operatorname{cotg} \varphi+\frac{p a^{2}}{2 \mathrm{~F}} \varphi\right] \\
+\rho_{1}\left[1+\rho_{2}(\lambda \varphi-\cos \varphi)\right]\left[\frac{\mathrm{A}}{\mathrm{F}} \cot \varphi_{1}+\frac{p a^{2}}{2 \mathrm{~F}} \varphi_{1}\right] \\
-\rho_{\varphi_{2}}\left[1+\rho_{1}(\lambda \varphi-\cos \varphi)\right]\left[\frac{\mathrm{A}}{\mathrm{F}} \operatorname{cotg} \varphi_{2}+\frac{p a^{2}}{2 \mathrm{~F}} \varphi_{2}\right]
\end{array}\right\}_{(3-40)}
$$

III.6.2. DÉtermination DE $f(\varphi)$.

Nous avons posé au chapitre III.2 :

$$
\mathrm{T}=q+f(\varphi) \quad \text { tel que } q=0 \quad \text { en } \varphi=\varphi_{1} \quad \text { et } \varphi=\varphi_{2}
$$

II s'ensuit :

et

$$
\begin{array}{ll}
f(\vartheta)=\mathrm{T}_{1} & \text { pour } \varphi=\vartheta_{1} \\
f(\varphi)=\mathrm{T}_{2} & \text { pour } \varphi=\varphi_{2}
\end{array}
$$

Soil en vertu de (3-13) :

$$
\begin{aligned}
& \frac{\mathrm{A}_{1}}{\lambda+\sin \varphi_{1}}+\mathrm{A}_{2} \frac{\lambda \varphi_{1}-\cos \varphi_{1}}{\lambda+\sin \varphi_{1}}=\mathrm{T}_{1} \\
& \frac{\mathrm{A}_{1}}{\lambda+\sin \varphi_{2}}+\mathrm{A}_{2} \frac{\lambda \varphi_{2}-\cos \varphi_{2}}{\lambda+\sin \varphi_{2}}=\mathrm{T}_{2}
\end{aligned}
$$


On en déduit :

$$
\begin{aligned}
& A_{1}=\frac{1}{\rho_{1}-\rho_{2}}\left[T_{1} \rho_{1}\left(\lambda+\sin \varphi_{1}\right)-T_{2} \rho_{2}\left(\lambda+\sin \varphi_{2}\right)\right] \\
& A_{2}=\frac{\rho_{1} \rho_{2}}{\rho_{1}-\rho_{2}}\left[T_{1}\left(\lambda+\sin \varphi_{1}\right)-T_{2}\left(\lambda+\sin \varphi_{2}\right)\right]
\end{aligned}
$$

D'où :

$$
f(\varphi)=\frac{1}{\left(\rho_{1}-\rho_{2}\right)(\lambda+\sin \varphi)}\left\{\begin{array}{l}
\mathrm{T}_{1 \rho_{1}}\left(\lambda+\sin \varphi_{1}\right)-\mathrm{T}_{2 \rho_{2}}\left(\lambda+\sin \varphi_{2}\right) \\
+\rho_{1} \rho_{2}\left[\mathrm{~T}_{1}\left(\lambda+\sin \varphi_{1}\right)-\mathrm{T}_{2}\left(\lambda+\sin \varphi_{2}\right)\right](\lambda \varphi-\cos \varphi)
\end{array}\right\}
$$

III.6.3. VALEUR DE $f(\varphi)-\Phi(p, \varphi)=\mathscr{F}(\varphi)$

(3-40) et (3-45) donnent :

$$
\begin{aligned}
& \mathscr{F}(\varphi)=\frac{1}{\left(\rho_{1}-\varphi_{2}\right)(\lambda+\sin \varphi)} \\
& \times\left[\begin{array}{l}
\rho_{2}\left[\lambda \frac{\mathrm{A}}{\mathrm{F}} \operatorname{cotg} \varphi_{2}+\lambda \frac{p a^{2}}{2 \mathrm{~F}} \varphi_{2}-\left(\lambda+\sin \varphi_{2}\right) \mathrm{T}_{2}\right]-\rho_{1}\left[\lambda \frac{\mathrm{A}}{\mathrm{F}} \operatorname{cotg} \varphi_{1}+\lambda \frac{p a^{2}}{2 \mathrm{~F}} \varphi_{1}-\left(\lambda+\sin \varphi_{1}\right) \mathrm{T}_{1}\right] \\
+\rho_{1 i_{2}}\left(\lambda \frac{\mathrm{A}}{\mathrm{F}} \operatorname{cotg} \varphi_{2}+\lambda \frac{p a^{2}}{2 \mathrm{~F}} \varphi_{2}-\left(\lambda+\sin \varphi_{2}\right) \mathrm{T}_{2}-\left[\lambda \frac{\mathrm{A}}{\mathrm{F}} \operatorname{cotg} \varphi_{1}+\lambda \frac{p a^{2}}{2 \mathrm{~F}} \varphi_{1}-\left(\lambda+\sin \varphi_{1}\right) \mathrm{T}_{1}\right]\right\}\left(\lambda \varphi_{-}-\cos \varphi_{1}\right) \\
+\lambda\left(\varphi_{1}-\rho_{2}\right)\left(\frac{\mathrm{A}}{\mathrm{F}} \operatorname{cotg} \varphi+\frac{p a^{2}}{2 \mathrm{~F}} \varphi\right)
\end{array}\right]
\end{aligned}
$$

Soit, en posant :

$$
\begin{aligned}
\mathrm{C}_{1} & =\lambda \frac{\mathrm{A}}{\mathrm{F}} \operatorname{cotg} \varphi_{1}+\lambda \frac{p a^{2}}{2 \mathrm{~F}} \varphi_{1}-\left(\lambda+\sin \varphi_{1}\right) \mathrm{T}_{1} \\
\mathrm{C}_{2} & =\lambda \frac{\mathrm{A}}{\mathrm{F}} \operatorname{cotg} \varphi_{2}+\lambda \frac{p a^{2}}{2 \mathrm{~F}} \varphi_{2}-\left(\lambda+\sin \varphi_{2}\right) \mathrm{T}_{2} \\
\frac{\mathrm{A}}{\mathrm{F}} & =\beta \\
\frac{p a^{2}}{2 \mathrm{~F}} & =\bar{\omega}
\end{aligned}
$$

$\mathfrak{J}(\varphi)=\frac{1}{\left(\rho_{1}-\rho_{2}\right)(\lambda+\sin \varphi)}\left[\rho_{2} C_{2}-\rho_{1} C_{1}+\rho_{1 \rho_{2}}\left(C_{2}-C_{1}\right)(\lambda \varphi-\cos \varphi)+\lambda\left(\rho_{1}-\rho_{2}\right)(\beta \operatorname{cotg} \varphi+\bar{\omega} \varphi)\right]$

II1.6.4. Détiermination DE $g(\varphi)$.

Nous avons posé :

$$
\mathrm{V}=u+g(\wp)
$$

tel que

$$
u^{\prime}+\nu \frac{\cos \varphi}{\lambda+\sin \varphi} u=0 \quad \text { en } \varphi_{1} \quad \text { et } \varphi_{2}
$$

Or :

$$
\mathrm{M}=-\frac{\mathrm{D}}{a}\left(y^{\prime}+\nu \frac{\cos \varphi}{\lambda+\sin \varphi} \eta\right)
$$


Soit :

$$
\mathrm{M}=-\mathrm{F}\left(\mathrm{V}^{\prime}+\nu \frac{\cos \varphi}{\lambda+\sin \varphi} \mathrm{V}\right) \text { puisque } \mathrm{V}=\frac{\mathrm{D}}{a \mathrm{~F}} y
$$

Nous aurons done :

$$
\begin{aligned}
& \mathrm{M}_{1}=-\mathrm{F}\left[g^{\prime}\left(\varphi_{1}\right)+v \frac{\cos \varphi_{1}}{\lambda+\sin \varphi_{1}} g\left(\varphi_{1}\right)\right] \\
& \mathrm{M}_{2}=-\mathrm{F}\left[g^{\prime}\left(\varphi_{2}\right)+v \frac{\cos \varphi_{2}}{\lambda+\sin \varphi_{2}} g\left(\varphi_{2}\right)\right]
\end{aligned}
$$

Compte tenu de (3-14), ces expressions s'écrivent:

$$
\begin{aligned}
& m_{1}=\frac{\mathrm{M}_{1}}{\mathrm{~F}}=\mathrm{B}_{1} \frac{(1-v) \cos \varphi_{1}}{\left(\lambda+\sin \varphi_{1}\right)^{2}}-\mathrm{B}_{2}\left[1-\frac{(1-v)\left(\lambda_{1}-\cos \varphi_{1}\right) \cos \varphi_{1}}{\left(\lambda+\sin \varphi_{1}\right)^{2}}\right] \\
& m_{2}=\frac{\mathrm{M}_{2}}{\mathrm{~F}}=\mathrm{B}_{1} \frac{(1-v) \cos \varphi_{2}}{\left(\lambda+\sin \varphi_{2}\right)^{2}}-\mathrm{B}_{2}\left[1-\frac{(1-\nu)\left(\lambda_{\varphi_{2}}-\cos \varphi_{2}\right) \cos \varphi_{2}}{\left(\lambda+\sin \varphi_{2}\right)^{2}}\right]
\end{aligned}
$$

ou encore en fonction de $q_{1} q_{2} i_{1}$ et $\mu_{2}$ :

$$
\begin{aligned}
& m_{1}\left(\frac{1}{\mu_{1}}-\frac{1}{\rho_{1}}\right)=B_{1}-\frac{B_{2}}{\mu_{1}} \\
& m_{2}\left(\frac{1}{\mu_{2}}-\frac{1}{\rho_{2}}\right)=B_{1}-\frac{B_{2}}{\mu_{2}}
\end{aligned}
$$

et inversement :

$$
\begin{aligned}
& \mathrm{B}_{1}=\frac{1}{\mu_{1}-\mu_{2}}\left[m_{1}\left(1-\frac{\mu_{1}}{\rho_{1}}\right)-m_{2}\left(1-\frac{\mu_{2}}{\rho_{2}}\right)\right] \\
& \mathrm{B}_{2}=\frac{1}{\mu_{1}-\mu_{2}}\left[m_{1 \mu_{2}}\left(1-\frac{\mu_{1}}{\rho_{1}}\right)-m_{2 \mu_{1}}\left(1-\frac{\mu_{2}}{\rho_{2}}\right)\right]
\end{aligned}
$$

Finalement :

$g(\varphi)=\frac{1}{\left(\mu_{1}-\mu_{2}\right)(\lambda+\sin \varphi)}\left\{\begin{array}{l}m_{1}\left(1-\frac{\mu_{1}}{\rho_{1}}\right)-m_{\cdot}\left(1-\frac{\mu_{2}}{\rho_{2}}\right) \\ +\left[m_{1 \mu_{2}}\left(1-\frac{\mu_{1}}{\rho_{1}}\right)-m_{2 \mu_{1}}\left(1-\frac{\mu_{2}}{\rho_{2}}\right)\right] \cdot(\lambda \varphi-\cos \varphi)\end{array}\right\}$

Si la rotation est nulle $(\mathrm{V}=0)$ sur la base $\varphi_{i}=\varphi_{2}$ par exemple, la condition $(3-53)$ est remplacée par :

$$
g\left(\%_{2}\right)=0
$$

donc :

$$
\mathrm{B}_{1}-\frac{\mathrm{B}_{2}}{P_{2}}=0
$$

ce qui revient à remplacer $i_{2}$ par $\oint_{2}$ dans l'équation (3-57) et par conséquent dans l'équation générale $(3-60)$.

De même, si la rotation est nulle en $\varphi=\varphi_{1}$ il suffit de remplacer dans $(3-60) \mu_{1}$ par $\rho_{1}$. 


\section{III.7. Valeurs des différentes constantes des seconds membres pour les six cas de charges envisagés :}

L'équation d'équilibre (2-1) intégrée par rapport à $\varphi$, s'écrit :

$r_{0} \mathrm{~N} \sin \varphi+r_{0} Q \cos \varphi=\mathrm{A}+\frac{p a^{2}}{2}(2 \lambda+\sin \varphi) \sin \varphi$

soit, puisque :

$$
\underset{\mathrm{F}}{r_{0} \mathrm{Q}}=\mathrm{T} \cdot \sin \varphi
$$

$\frac{r_{0} \mathrm{~N}}{\mathrm{~F}} \sin \varphi+\mathrm{T} \sin \varphi \cos \varphi=\beta+\bar{\omega}(2 \lambda+\sin \varphi) \sin \varphi$

Appelons $W_{1}$ et $W_{2}$ les efforts axiaux appliqués respectivement aux bases 1 et 2 .

$r_{01} \mathrm{~W}_{1}=r_{01} \mathrm{~N}_{1} \sin \varphi_{1}+r_{01} \mathrm{Q}_{1} \cos \varphi_{1}$

$r_{02} \mathrm{~W}_{2}=r_{02} \mathrm{~N}_{2} \sin \varphi_{2}+r_{02} \mathrm{Q}_{2} \cos \varphi_{2}$

ou

$$
\begin{aligned}
& \mathrm{N}_{i}=\mathrm{W}_{i} \sin \varphi_{i} \\
& \mathrm{Q}_{i}=\mathrm{W}_{i} \cos \varphi_{i}
\end{aligned}
$$

L'équation (3-61) s'écrit encore :

$$
r_{0 i} \mathrm{~W}_{i}=\mathrm{A}+\frac{p a^{2}}{2}\left(2 \lambda+\sin \varphi_{i}\right) \sin \vartheta_{i}(3-66)
$$

et l'on obtient :

$$
\mathrm{T}_{i}=\frac{r_{0 i} \mathrm{~W}_{i}}{\mathrm{~F}} \operatorname{cotg} \varphi_{i}
$$

$i=1$ ou 2 suivant la base considérée.

Si l'on désigne par $\mathrm{H}_{1}$ et $\mathrm{H}_{2}$ les efforts radiaux appliqués respectivement aux bases 1 et 2,

on a :

$\mathrm{H}_{i} \cos \varphi_{i}=\mathrm{N}_{i} \quad-\mathrm{H}_{i} \sin \varphi_{i}=Q_{i} \quad i=1$ ou 2

Premier cas. - Effort radial $\mathrm{H}_{1}$ appliqué à la base 1 .

On a :

$$
\mathrm{A}=0 \quad p=0 \quad \text { soit } \beta=\bar{\omega}=0
$$

L'équation (3-62) donne:

$$
\frac{r_{01} H_{1}}{\mathrm{~F}} \sin \varphi_{1} \cos \varphi_{1}+\mathrm{T}_{1} \sin \varphi_{1} \cos \varphi_{1}=0
$$

Nous poserons :

$$
\mathrm{F}=-r_{01} \mathrm{H}_{1}=-a\left(\lambda+\sin \varphi_{1}\right) \mathrm{H}_{1}
$$

On en déduit :

$$
\mathrm{T}_{1}=1
$$

D'autre part :

$$
\mathrm{T}_{2}=\frac{r_{02} \mathrm{Q}_{2}}{\mathrm{~F} \sin \varphi_{2}}=0
$$

Les moments M s'annulent sur les bases :

$$
m_{1}=m_{2}=0 \quad g(\varphi) \equiv 0
$$

Les constantes $\mathrm{C}_{1}$ et $\mathrm{C}_{22}$ de $\mathscr{G}(\varphi)$ ont donc pour valeurs

$$
\begin{aligned}
& \mathrm{C}_{1}=-\left(\lambda+\sin \varphi_{1}\right) \\
& \mathrm{C}_{2}=0
\end{aligned}
$$

Deuxième cas. - Effort radial $\mathrm{H}_{2}$ appliqué à la base 2 .

On a :

$$
\mathrm{A}=0 \quad p=0 \quad \text { soit } \beta=\bar{\omega}=0
$$

On obtient en posant :

$$
\begin{aligned}
& \mathrm{F}=-r_{02} \mathrm{H}_{2}=-a\left(\lambda+\sin \varphi_{.2}\right) \mathrm{H}_{2} \\
& \mathrm{~T}_{2}=1 \quad \mathrm{~T}=0 \\
& m_{1}=m_{2}=0 \quad g(\varphi) \equiv 0 \\
& \mathrm{C}_{1}=0 \\
& \mathrm{C}_{2}=-\left(\lambda+\sin \varphi_{2}\right)
\end{aligned}
$$

Troisième cas. - Efrorts axiaux équilibrés sur les deux bases.

Soient $W_{1}$ et $W_{2}$ ces efforts.

La relation $(3-66)$ donne avec $\bar{\omega}=0$

$$
r_{01} \mathrm{~W}_{1}=r_{02} \mathrm{~W}_{2}=\mathrm{A}
$$

Posons :

$$
\begin{aligned}
& \mathrm{F}=r_{01} \mathrm{~W}_{1}=a\left(\lambda+\sin \varphi_{1}\right) \mathrm{W}_{1}=\mathrm{A} \\
& \mathrm{T}_{1}=\frac{r_{01} \mathrm{Q}_{1}}{\mathrm{~F} \sin \varphi_{1}}=\operatorname{cotg} \varphi_{1} \\
& \mathrm{~T}_{2}=\frac{r_{02} \mathrm{Q}_{2}}{\mathrm{~F} \sin \varphi_{2}}=\operatorname{cotg} \varphi_{2}
\end{aligned}
$$

Les constantes $\mathrm{C}_{1}$ et $\mathrm{C}_{2}$ s'expriment done par :

$$
\begin{aligned}
& \mathrm{C}_{1}=-\cos \varphi_{1} \\
& \mathrm{C}_{2}=-\cos \varphi_{2}
\end{aligned}
$$


D'autre part :

$$
\beta=\frac{\mathrm{A}}{\mathrm{F}}=1 \quad m_{1}=m_{2}=0 \quad g(\varphi) \equiv 0
$$

Quatrième cas. -- Pression équilibrée par un effort axial $W_{1}$ sur la base 1 .

Posons

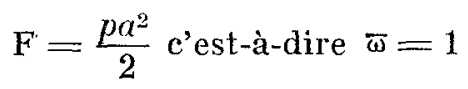

La relation (3-66) donne :

$$
A=-\frac{p a^{2}}{2}\left(2 \lambda+\sin \varphi_{2}\right) \sin \varphi_{2}
$$

soit :

$$
\frac{A}{F}=\beta=-\left(2 \lambda+\sin \varphi_{2}\right) \sin \varphi_{2}
$$

et

$$
\frac{r_{01} \mathrm{~W}_{1}}{\mathrm{~F}}=\frac{\mathrm{A}}{\mathrm{F}}+\left(2 \lambda+\sin \varphi_{1}\right) \sin \varphi_{1}
$$

Or :

$$
\begin{aligned}
& \mathrm{T}_{1}=\frac{r_{01} \mathrm{Q}_{1}}{\mathrm{~F} \sin \varphi_{1}}=\frac{r_{01} \mathrm{~W}_{1}}{\mathrm{~F}} \operatorname{cotg} \varphi_{1} \\
& \mathrm{~T}_{1}=\frac{\mathrm{A}}{\mathrm{F}} \operatorname{cotg} \varphi_{1}+\left(2 \lambda+\sin \varphi_{1}\right) \cos \varphi_{1}
\end{aligned}
$$

D'autre part, $\mathrm{T}_{2}=0$
D'où :

$$
\begin{aligned}
C_{1}= & \left(2 \lambda+\sin \varphi_{2}\right) \sin \varphi_{2} \cos \varphi_{1}- \\
& \left(2 \lambda+\sin \varphi_{1}\right)\left(\lambda+\sin \varphi_{1}\right) \cos \varphi_{1}+\lambda \varphi_{1} \\
C_{2}= & \lambda\left[\varphi_{2}-\left(2 \lambda+\sin \varphi_{2}\right) \cos \varphi_{2}\right]
\end{aligned}
$$

En outre :

$$
m_{1}=m_{2}=0 \quad g(\varphi) \equiv 0
$$

Cinquieme cas. - Moment $\mathbf{M}_{1}$ agissant sur la base 1 .

Posons :

$$
\mathrm{F}=\mathrm{M}_{1} \quad \text { soit } m_{1}=1
$$

On a :

$$
A=\beta=\bar{\omega}=m_{2}=0
$$

et d'autre part :

$$
\mathscr{H}(\varphi) \equiv 0
$$

Sixième cas. - Moment $\mathrm{M}_{2}$ agissant sur la base 2 .

Posons :

$$
\mathrm{F}=\mathrm{M}_{2} \quad \text { soit } m_{2}=1
$$

On a :

$$
A=\beta=\bar{\omega}=m_{1}=0
$$

et

$$
\mathscr{F}(\varphi) \equiv 0
$$

\section{IV. - RESOLUTION DES EQUATIONS INTEGRALES}

$$
\begin{array}{r}
\mathrm{T}(\varphi)=\int_{\varphi_{1}}^{\varphi_{2}} \mathrm{~K}_{1}(\varphi, \alpha)[(1+\nu) \mathrm{T}(\alpha) \sin \alpha-k \mathrm{~V}(\alpha) \sin \alpha] d \alpha \\
+\mathscr{F}(\vartheta) \\
\mathrm{V}(\varphi)=\int_{\varphi_{1}}^{\varphi_{2}} \mathrm{~K}_{2}(\varphi, \alpha)[(1-\nu) \mathrm{V}(\alpha) \sin \alpha+\mathrm{T}(\alpha) \sin \alpha] d \alpha \\
+g(\varphi)
\end{array}
$$

avec :

$$
\begin{aligned}
& \mathrm{V}(\varphi)=\frac{\mathrm{D}}{a \mathrm{~F}} y(\varphi) \\
& \mathrm{T}(\varphi)=\frac{r_{0}(\varphi) \mathrm{Q}(\varphi)}{\mathrm{F} \sin \varphi}
\end{aligned}
$$

\section{IV.1. Transformation des équations.}

Pour éviter le pôle $\sin \varphi=0$, nous poserons comme nouvelle variable pour le cisaillement

$$
\mathrm{U}(\varphi)=\mathrm{T}(\varphi) \sin \varphi=\frac{r_{0} \mathrm{Q}}{\mathrm{F}}
$$

et d'une façon générale

$$
\begin{gathered}
Z(\varphi)=(1+\nu) \mathrm{U}(\varphi)-k V(\varphi) \sin \varphi \\
\mathrm{Y}(\varphi)=\mathrm{U}(\varphi)+(1-\nu) \mathrm{V}(\varphi) \sin \varphi
\end{gathered}
$$


Inversement :

$$
\begin{aligned}
& U(\varphi)=\frac{k V(\varphi)+(1-\nu) Z(\varphi)}{\delta \sin \varphi} \\
& V(\varphi)=\frac{(1+\nu) Y(\varphi)-Z(\varphi)}{\delta \sin \varphi}
\end{aligned}
$$

avec :

$$
\delta=1-v^{2}+k
$$

Les équations (4-1) et (4-2) s'écrivent alors :

$$
\begin{aligned}
\mathrm{T}(\varphi) & =\frac{U(\varphi)}{\sin \varphi}=\frac{k \mathrm{Y}+(1-v) Z}{\delta \sin \varphi} \\
& =\int_{\varphi_{1}}^{\varphi_{2}} \mathrm{~K}_{1}(\varphi, \alpha) Z(\alpha) d \alpha+\mathscr{F}(\varphi) \\
V(\varphi) & =\frac{(1+\nu) \mathrm{Y}-Z}{\delta \sin \varphi} \\
& =\int_{\varphi_{1}}^{\varphi_{2}} \mathrm{~K}_{2}(\varphi, \alpha) \mathrm{Y}(\alpha) d \alpha+g(\varphi)
\end{aligned}
$$

Multiplions les deux membres de (4-9) par $\left(\vartheta_{1}-\rho_{2}\right) \sin \varphi$ et les deux membres de (4-10) par $\left(u_{1}-u_{2}\right) \sin \varphi$ et posons :

$$
\begin{aligned}
& A_{1}=\frac{\rho_{1}-\rho_{2}}{\delta} k \\
& A_{2}=\frac{\rho_{1}-\rho_{2}}{\delta}(1-\nu) \\
& A_{3}=\frac{\mu_{1}-\mu_{2}}{\delta}(1+\nu) \\
& A_{1}=\frac{\mu_{1}-\mu_{2}}{\delta}
\end{aligned}
$$

$$
\begin{aligned}
& \mathrm{K}(\varphi, \alpha)=\left(\varphi_{1}-\varphi_{2}\right) \mathbf{K}_{1}(\varphi, \alpha) \sin \varphi \\
& \mathrm{H}(\varphi, \alpha)=\left(\mu_{1}-\mu_{2}\right) \mathrm{K}_{2}(\varphi, \alpha) \sin \varphi \\
& \mathrm{F}(\varphi)=\left(\varphi_{1}-\varphi_{2}\right) g(\varphi) \sin \varphi \\
& \mathrm{G}(\varphi)=\left(\mu_{1}-\mu_{2}\right) g(\varphi) \sin \varphi
\end{aligned}
$$

Ces équations deviennent :

$A_{1} Y+A_{2} Z-\int_{\varphi_{1}}^{\varphi_{2}} K(\varphi, \alpha) Z(\alpha) d \alpha=F(\varphi)$

$$
\mathrm{A}_{3} \mathrm{Y}+\mathrm{A}_{4} Z-\int_{\varphi_{1}}^{\varphi_{2}} \mathrm{H}(\varphi, \alpha) \mathrm{Y}(\alpha) d \alpha=\mathrm{G}(\varphi)
$$

\section{IV.2. Résolution numérique des équations (4-16) et (4-17).}

Pour résoudre le système des deux équations intégrales (4-16) et (4-17), nous remplacerons les intégrales par des sommes en utilisant la méthode de Simpson.

Soit $\varphi_{1}$ et $\varphi_{n}$ les points extrêmes limitant l'arc de cercle méridien étudié.

Considérons sur cet arc $n$ points définissant $n-1$ intervalles égaux.

L’application de la méthode de Simpson nécessite un nombre d'intervalles pair, $n$ doit donc ètre un nombre impair au moins égal à 3 .

Soit :

$$
\Delta \varphi=\frac{\varphi_{n}-\varphi_{1}}{n-1}
$$

L'intégrale de (4-16) par exemple se calcule de la façon suivante :

$$
\begin{aligned}
& \int_{\varphi}^{\varphi_{\alpha}} \mathrm{K}(\varphi, \alpha) Z(\alpha) d \alpha \\
& \quad \cong \frac{\Delta \varphi}{3}\left[\begin{array}{l}
\mathrm{K}\left(\varphi, \varphi_{1}\right) Z\left(\varphi_{1}\right) \\
+4 \mathrm{~K}\left(\varphi, \varphi_{2}\right) Z\left(\varphi_{2}\right)+2 \mathrm{~K}\left(\varphi_{,} \varphi_{3}\right) Z\left(\varphi_{3}\right) \\
+4 \mathrm{~K}\left(\varphi, \varphi_{4}\right) Z\left(\varphi_{4}\right) \ldots+\mathrm{K}\left(\varphi, \varphi_{n}\right) Z\left(\varphi_{n}\right)
\end{array}\right]
\end{aligned}
$$

Soit, en posant :

$$
\Delta k=\eta_{k} \frac{\Delta \varphi}{3}
$$

$\int_{\varphi_{1}}^{\varphi_{2}} \mathrm{~K}(\varphi, \alpha) Z(\alpha) d \alpha \cong \sum_{k=1}^{n} \mathrm{~K}\left(\varphi, \varphi_{k}\right) Z\left(\varphi_{k}\right) \Delta_{k}$

$r_{i k}$ est un coefficient numérique défini en fonction de $k$ par la correspondance suivante:

$$
\begin{aligned}
& \eta_{k}=1 \quad 4 \quad 2 \quad 4 \quad 2 \quad \ldots \quad 4 \quad 1 \\
& \text { pour } k=1 \quad 2 \quad 3 \quad 4 \quad 5 \quad \ldots \quad n-1 \quad n(4-22)
\end{aligned}
$$


Les équations (4-16) et (4-17) se ramènent à un système de $2 n$ équations linéaires à $2 n$ inconnues :

$$
\begin{aligned}
& \mathrm{A}_{1} \mathrm{Y}_{i}+\mathrm{A}_{2} \mathrm{Z}_{i}-\sum_{k=1}^{n} \mathrm{~K}_{i k} Z_{k} \Delta_{k}={ }_{j} \mathbf{F}_{i} \\
& \mathrm{~A}_{3} \mathrm{Y}_{i}+\mathrm{A}_{\mathbf{4}} Z_{i}-\sum_{k=1}^{n} \mathrm{H}_{i k} \mathbf{Y}_{k} \Delta_{k}={ }_{j} \mathrm{G}_{i}
\end{aligned}
$$

où

$$
i=1 \quad 2 \quad 3 \quad \ldots \quad n
$$

$j$ est un indice caractérisant les seconds membres fonction du cas de charge étudié ( $j$ varie de 1 à 6 ).

D’une façon générale, nous avons posé :

$$
\begin{aligned}
\mathbf{Y}_{i} & =\mathbf{Y}\left(\varphi_{i}\right) & \mathrm{F}_{i} & =\mathbf{F}\left(\varphi_{i}\right) \ldots \\
\mathbf{Y}_{k} & =\mathbf{Y}\left(\varphi_{k}\right) & \mathrm{K}_{i k} & =\mathrm{K}\left(\varphi_{i}, \varphi_{k}\right) \ldots
\end{aligned}
$$

Lat résolution du système (4-23), (4-24) donne $n$ valeurs de $Y_{i}$ et $n$ valeurs de $Z_{i}$ pour chacun des six cas de charges envisagés.

On en déduit ensuite les valeurs de $\mathrm{U}$ et $\mathrm{V}$ à l'aide des formules (4-6) et (4-7).

Dans les calculs, nous éviterons le point $\varphi=0$, sauf pour le premier point ô considéré. Dans ce cas, la formule (4-7) n'est plus utilisable mais on peut calculer $\mathrm{V}\left(\sigma_{1}\right)=\mathrm{V}_{1}$ de la façon suivante :
Considérons l'équation (4-10) :

$$
\begin{gathered}
\mathrm{V}(\varphi)=\int_{\varphi_{1}}^{\varphi_{2}} \mathbf{K}_{2}(\varphi, \alpha) \mathrm{Y}(\alpha) d \alpha+g(\varphi) \\
\mathrm{V}(\varphi)=\frac{1+\mu_{2}(\lambda \varphi-\cos \varphi)}{\left(\mu_{1}-\mu_{2}\right)(\lambda+\sin \varphi)} \\
\times \int_{\varphi_{1}}^{\varphi} \frac{1+\mu_{1}(\lambda \alpha-\cos \alpha)}{\lambda+\sin \alpha} \mathrm{Y}(\alpha) d \alpha+ \\
+\frac{1+\mu_{1}(\lambda \varphi-\cos \varphi)}{\left(\mu_{1}-\mu_{2}\right)(\lambda+\sin \varphi)} \\
\times \int_{\varphi}^{\varphi_{2}} \frac{1+\mu_{2}(\lambda \alpha-\cos \alpha)}{\lambda+\sin \alpha} \mathrm{Y}(\alpha) d \alpha+ \\
\frac{1}{\left(\mu_{1}-\mu_{2}\right)(\lambda+\sin \varphi)}\left\{m_{1}\left(1-\frac{\mu_{1}}{\rho_{1}}\right)-m_{2}\left(1-\frac{\mu_{2}}{\rho_{2}}\right)\right. \\
\left.+\left[m_{1} \mu_{2}\left(1-\mu_{1}\right)-m_{2 \mu_{1}}\left(1-\frac{\mu_{2}}{\rho_{2}}\right)\right](\lambda \varphi-\cos \varphi)\right\}
\end{gathered}
$$

qui donne pour $\varphi=\varphi_{1}$

$$
\begin{aligned}
V\left(\varphi_{1}\right)= & \frac{1-\left(\mu_{1} / \rho_{1}\right)}{\left(\mu_{1}-\mu_{2}\right)\left(\lambda+\sin \varphi_{1}\right)} \\
& \times\left[J+m_{1}\left(1-\frac{\mu_{2}}{\rho_{1}}\right)-m_{2}\left(1-\frac{\mu_{2}}{\rho_{2}}\right)\right] \\
(4-26) & \\
J= & \frac{1+\mu_{2}(\lambda \alpha-\cos \alpha)}{\lambda+\sin \alpha} \mathrm{Y}(\alpha) d \alpha
\end{aligned}
$$

peut se calculer numériquement en fonction des $\mathbf{Y}_{i}$

$$
\mathbf{J}=\sum_{k=1}^{n} \frac{1+\mu_{2}\left(\lambda \varphi_{k}-\cos \varphi_{k}\right)}{\lambda+\sin \varphi_{k}} \mathrm{Y}_{k} \Delta_{k}
$$

\section{V. - DETERMINATION DES COEFFICIENTS D'INFLUENCE}

\section{V.1. Calcul de $\frac{d V}{d \varphi}$}

Partant de (4-25) on obtient :

$$
\begin{aligned}
\left(\mu_{1}-\mu_{2}\right)(\lambda+\sin \varphi) \mathrm{V} & =\left[1+\mu_{2}(\lambda \varphi-\cos \varphi)\right] \int_{\varphi_{1}}^{\varphi} \frac{1+\mu_{1}(\lambda \alpha-\cos \alpha)}{\lambda+\sin \alpha} \mathrm{Y}(\alpha) d \alpha \\
& +\left[1+\mu_{1}(\lambda \varphi-\cos \varphi)\right] \int_{\varphi \cdot}^{\varphi_{2}} \frac{1+\mu_{2}(\lambda \alpha-\cos \alpha)}{\lambda+\sin \alpha} \mathrm{Y}(\alpha) d \alpha+m_{1}\left(1-\frac{\mu_{1}}{\rho_{1}}\right) \\
& -m_{2}\left(1-\frac{\mu_{2}}{\rho_{2}}\right)+\left[m_{1} \mu_{2}\left(1-\frac{\mu_{1}}{\rho_{1}}\right)-m_{2, \mu_{1}}\left(1-\frac{\mu_{2}}{\rho_{2}}\right)\right](\lambda \varphi-\cos \varphi)
\end{aligned}
$$

Dérivons par rapport à $\varphi$ et divisons les deux membres par $\lambda+\sin \varphi$ :

$$
\begin{aligned}
\left(\mu_{1}-\mu_{2}\right)\left(\mathrm{V}^{\prime}+\mathrm{V} \frac{\cos \varphi}{\lambda+\sin \varphi}\right) & =\mu_{2} \int_{\varphi_{1}}^{\varphi} \frac{1+\mu_{1}(\lambda \alpha-\cos \alpha)}{\lambda+\sin \alpha} \mathrm{V}(\alpha) d \alpha \\
& +\mu_{1} \int_{\alpha}^{\varphi_{2}} \frac{1+\mu_{2}(\lambda \alpha-\cos \alpha)}{\lambda+\sin \alpha} \mathrm{V}(\alpha) d \alpha+m_{1 \mu_{2}}\left(1-\frac{\mu_{1}}{\rho_{1}}\right)-m_{2} \mu_{1}\left(1-\frac{\mu_{2}}{\rho_{2}}\right)
\end{aligned}
$$


Ou encore :

$$
\begin{aligned}
\left(\mu_{1}-\mu_{2}\right)\left(V^{\prime}\right. & \left.+V \frac{\cos \varphi}{\lambda+\sin \varphi}\right) \\
& =-\left(\mu_{1}-\mu_{2}\right) \int_{\varphi_{1}}^{\varphi} \frac{\mathrm{V}(\alpha)}{\lambda+\sin \alpha} d \alpha \\
& +\mu_{1} \int_{\varphi_{1}}^{\varphi_{2}} \frac{1+\mu_{2}(\lambda \alpha-\cos \alpha)}{\lambda+\sin \alpha} \mathrm{V}(\alpha) d \alpha \\
& +m_{1 \mu_{2}}\left(1-\frac{\mu_{1}}{\rho_{1}}\right)-m_{2 \mu_{1}}\left(1-\frac{\mu_{2}}{\rho_{2}}\right)
\end{aligned}
$$

Compte tenu de (4-26) et en posant :

$$
J_{\varphi}=\int_{\varphi_{1}}^{\varphi} \frac{V(\alpha)}{\lambda+\sin \alpha} d \alpha
$$

on obtient finalement :

$$
\begin{aligned}
V^{\prime}=-V \frac{\cos \varphi}{\lambda+\sin \varphi}-J_{\varphi} & \\
& +(1-\nu) \frac{\cos \varphi_{1}}{\lambda+\sin \varphi_{1}} V_{1}-m_{1}
\end{aligned}
$$

\section{V.2. Calcul de $\frac{d \mathrm{~T}}{d \varphi}$}

La formule (4-9) s'écrit encore :

$$
\begin{aligned}
& T(\varphi)=\frac{1+\rho_{2}(\lambda \varphi-\cos \varphi)}{\left(\varphi_{1}-\rho_{2}\right)(\lambda+\sin \varphi)} \int_{\varphi_{1}}^{\varphi} \frac{\lambda \alpha-\cos \alpha}{\lambda+\sin \alpha} Z(\alpha) d \alpha \\
& +\frac{1+\rho_{1}(\lambda \varphi-\cos \varphi)}{\left(\rho_{1}-\rho_{2}\right)(\lambda+\sin \varphi)} \int_{\varphi}^{\varphi_{2}} \frac{\lambda \alpha-\cos \alpha}{\lambda+\sin \alpha} Z(\alpha) d \alpha+\mathscr{F}^{4}(\varphi)
\end{aligned}
$$

$$
\begin{aligned}
\mathscr{F}(\varphi) & =\frac{1}{\left(\rho_{1}-\rho_{2}\right)(\lambda+\sin \varphi)} \\
& \times\left[\begin{array}{r}
\rho_{2} C_{2}-\rho_{1} C_{1}+\rho_{1} \rho_{2}\left(C_{2}-C_{1}\right)(\lambda \varphi-\cos \varphi)^{-} \\
+\lambda\left(\rho_{1}-\rho_{2}\right)(\beta \operatorname{cotg} \varphi+\omega \varphi)
\end{array}\right]
\end{aligned}
$$

multiplions les deux membres de (5-6) par $\left(\rho_{1}-\rho_{2}\right)(\lambda+\sin \varphi)$.

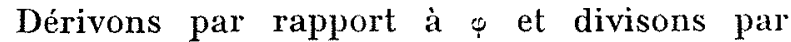
$(\lambda+\sin \varphi)$; nous obtenons :

$$
\begin{aligned}
& \left(\rho_{1}-\rho_{2}\right)\left(\mathbf{T}^{\prime}+\mathbf{T} \frac{\cos \varphi}{\lambda+\sin \varphi}\right) \\
& =\rho_{2} \int_{\psi_{1}}^{\varphi} \frac{1+\rho_{1}(\lambda \alpha-\cos \alpha)}{\lambda+\sin \alpha} Z(\alpha) d \alpha \\
& +\rho_{1} \int_{\varphi}^{\lambda_{2}} \frac{1+\rho_{2}(\lambda \alpha-\cos \alpha)}{\lambda+\sin \alpha} Z(\alpha) d \alpha+\rho_{1} \rho_{2}\left(C_{2}-C_{1}\right) \\
& +\lambda\left(\rho_{1}-\rho_{2}\right)\left(\frac{\bar{\omega}}{\lambda+\sin \varphi}-\frac{\beta}{(\lambda+\sin \varphi)\left(\sin ^{2} \varphi\right)}\right)
\end{aligned}
$$

ou encore en posant :

$$
I=\int_{\varphi_{1}}^{\varphi_{2}} \frac{1+\varphi_{2}(\lambda \alpha-\cos \alpha)}{\lambda+\sin \alpha} Z(x) d \alpha
$$

et

$$
\begin{gathered}
\mathrm{I}_{\varphi}=\int_{\varphi_{1}}^{\varphi} \frac{Z(\alpha)}{\lambda+\sin \alpha} d \alpha \\
\mathrm{T}^{\prime}=-\mathrm{T} \frac{\cos \varphi}{\lambda+\sin \varphi}-\mathrm{I}_{\varphi}+\frac{\rho_{1}}{\rho_{1}-\rho_{2}} \mathrm{I} \\
+\frac{\rho_{1} \rho_{2}}{\rho_{1}-\rho_{2}}\left(\mathrm{C}_{2}-\mathrm{C}_{1}\right) \\
+\lambda\left(\frac{\bar{\omega}}{\lambda+\sin \varphi}--\frac{\beta}{\left(\lambda+\frac{\beta}{\sin \varphi)\left(\sin ^{2} \varphi\right)}\right)}\right.
\end{gathered}
$$

\section{V.3. Détermination des efforts.}

\section{V.3.1. EFfort NonMal N.}

On a, d'une facon générale, d'après la formule $(2-27)$ :

$$
\mathrm{N}=\frac{\mathrm{A}+\mathrm{S}}{r_{0} \sin \varphi}-\frac{x \cos \varphi}{r_{0}}
$$

Soit :

$$
\begin{aligned}
& N=\frac{1}{a(\lambda+\sin \varphi)} \\
& \times\left[\frac{\mathrm{A}}{\sin \varphi}+\frac{p a^{2}}{2}(2 \lambda+\sin \varphi)-x \cos \varphi\right]
\end{aligned}
$$


et :

$\frac{a N}{F}=\frac{\beta}{\sin \varphi(\lambda+\sin \varphi)}+\bar{\omega} \frac{2 \lambda+\sin \varphi}{\lambda+\sin \varphi}-\frac{T \cos \varphi}{\lambda+\sin \varphi}$

ou encore :

$\frac{a N}{F}=\frac{\beta}{\sin \varphi(\lambda+\sin \varphi)}+\bar{\omega} \frac{2 \lambda+\sin \varphi}{\lambda+\sin \varphi}-\frac{U \operatorname{cotg} \varphi}{\lambda+\sin \varphi}$

La formule précédente permet en général de calculer la valeur de $\frac{a N}{F}$. Elle est en défaut pour $\varphi=k \pi$.

Nous éviterons donc de choisir ce point dans les calculs numériques sauf pour les bornes $\varphi_{1}$ et $\varphi_{2}$. Dans ces deux cas, nous pouvons déterminer les vraies valeurs de $\frac{a N}{F}$ suivant les cas de charges.

On trouve ainsi facilement les résultats suivants :

Premier cas. - Effort radial $\mathrm{H}_{1}$ :

$$
\begin{array}{ll}
\varphi=\varphi_{1} \quad \frac{a N_{1}}{F}=-\frac{\cos \varphi_{1}}{\lambda+\sin \varphi_{1}} \\
\varphi=\varphi_{2} \quad \frac{a N_{2}}{F}=0
\end{array}
$$

Deuxième cas. — Effort radial $\mathrm{H}_{2}$ :

$$
\begin{array}{ll}
\varphi=\varphi_{1} & \frac{a \mathrm{~N}_{1}}{\mathrm{~F}}=0 \\
\varphi=\varphi_{2} & \frac{a \mathrm{~N}_{2}}{\mathrm{~F}}=-\frac{\cos \varphi_{2}}{\lambda+\sin \varphi_{2}}
\end{array}
$$

Troisième cas. - Efforts axiaux équilibrés :

$$
\begin{aligned}
& \varphi=\varphi_{1} \quad \frac{a N_{1}}{F}=\frac{\sin \varphi_{1}}{\lambda+\sin \varphi_{1}} \\
& \varphi=\varphi_{2} \cdot \frac{a N_{2}}{F}=\frac{\sin \varphi_{2}}{\lambda+\sin \varphi_{2}}
\end{aligned}
$$

Quatrième cas. - Pression équilibrée par un effort axial en 1 :

$$
\begin{gathered}
\varphi=\varphi_{1} \quad \frac{a N_{1}}{\mathrm{~F}} \frac{\sin \varphi_{1}}{\lambda+\sin \varphi_{1}} \\
\times\left[\left(2 \lambda+\sin \varphi_{1}\right) \sin \varphi_{1}-\left(2 \lambda+\sin \varphi_{2}\right) \sin \varphi_{2}\right] \\
\varphi=\varphi_{2} \quad \frac{a N_{2}}{\mathrm{~F}}=0
\end{gathered}
$$

Cinquième cas. - Moment $\mathrm{M}_{1}$ :

$$
\begin{array}{ll}
\varphi=\vartheta_{1} & \frac{a N_{1}}{\mathrm{~F}}=0 \\
\varphi=\vartheta_{2} & \frac{a N_{2}}{\mathrm{~F}}=0
\end{array}
$$

Sixième cas. - Moment $\mathrm{M}_{2}$ :

$$
\begin{array}{ll}
\vartheta=\varphi_{1} & \frac{a N_{1}}{\mathrm{~F}}=0 \\
p=\varphi_{2} & \frac{a N_{2}}{\mathrm{~F}}=0
\end{array}
$$

V.3.2. EFFort NORMAL $n$ D'APRÈs (2-28).

$$
n=-\frac{1}{a} \frac{d x}{d \varphi}-\frac{\mathrm{A}}{a \sin ^{2} \varphi}+\frac{p a^{2}}{2}
$$

soit :

$$
\frac{a n}{\mathrm{~F}}=-\mathrm{T}^{\prime}-\frac{\beta}{\sin ^{2} \varphi}+\bar{\omega}
$$

En combinant les relations (5-10), (5-11) et (5-13), on obtient :

$$
\begin{aligned}
& \underset{\mathrm{F}}{a n}=-\frac{a \mathrm{~N}}{\mathrm{~F}}+\mathrm{I}_{\varphi}-\frac{p_{1}}{p_{1}-p_{2}} \\
& x\left[I+\rho_{2}\left(C_{2}-C_{1}\right)\right]+2 \bar{\omega}
\end{aligned}
$$

\section{V.3.3. Cisaillement Q.}

Par définition nous avons posé :

d'où :

$$
\mathrm{U}=\frac{r_{0} \mathrm{Q}}{\mathrm{F}}
$$

$$
\frac{a \mathrm{Q}}{\mathrm{F}}=\frac{\mathrm{U}}{\lambda+\sin \varphi}
$$

\section{V.4. Détermination des moments.}

Ils s'expriment en fonction de $\mathrm{V}$ et $\mathrm{V}^{\prime}$ déterminés au paragraphe $\mathrm{V}-1$ formule (5-5) par :

$$
\begin{aligned}
& \frac{M}{F}=-\left(V^{\prime}+\nu \frac{\cos \varphi}{\lambda+\sin \varphi} V\right) \\
& \frac{m}{F}=-\left(\nu V^{\prime}+\frac{\cos \varphi}{\lambda+\sin \varphi} V\right)
\end{aligned}
$$




\section{V.5. Dilatation radiale.}

Elle est définie par :

$$
d=r_{0} \varepsilon_{\theta}=v \cos \varphi--w \sin \varphi
$$

ou encore, en fonction de $n$ et $\mathrm{N}$ :

$$
\begin{gathered}
d=\frac{r_{0}}{\mathrm{E} e}(n-\nu \mathrm{N}) \\
d=\frac{a(\lambda+\sin \varphi)}{\mathrm{E} e}(n--\vee \mathrm{N})
\end{gathered}
$$

\section{V.6. Flèche axiale.}

Elle est donnée par :

$$
f=v \sin \varphi+w \cos \varphi
$$

Dérivons par rapport à $\emptyset$ :

$$
f^{\prime}=\left(v^{\prime}-w\right) \sin \varphi+\left(v+w^{\prime}\right) \cos \varphi(5-22)
$$

Or :

$$
v^{\prime}-w=\frac{a}{\mathrm{E} e}(\mathrm{~N}-\mathrm{v} n)
$$

et :

$$
v+w^{\prime}=a y
$$

d'où :

$$
f^{\prime}=\frac{a}{\mathrm{E} c}(\mathrm{~N}-\nu n) \sin \varphi+a y \cos \varphi
$$

En supposant la flèche axiale nulle en $\varphi=\varphi_{1}$. $f=\int_{\varphi_{1}}^{\varphi}\left[\frac{a}{\mathrm{E} e}(\mathrm{~N}-\nu n) \sin \varphi+a y \cos \varphi\right] d \varphi$

\section{V.7. Définition des contraintes.}

On a d'une façon générale

$$
\begin{aligned}
\sigma_{u} & =\frac{n}{e} & \sigma_{\mathrm{M}} & =\frac{6 \mathrm{M}}{e^{2}} \\
\sigma_{\mathrm{N}} & =\frac{\mathrm{N}}{e} & \sigma_{m} & =\frac{6 \mathrm{~m}}{e^{2}} \\
\sigma_{\mathrm{Q}} & =\frac{3}{2} \frac{\mathrm{Q}}{e} & y & =\frac{a \mathrm{~F}}{\mathrm{D}} \quad \mathrm{V}
\end{aligned}
$$

Nous définirons pour chacun des cas de charge une contrainte $\sigma_{\mathrm{F}}$ de référence.
Premier cas. - Effort radial $\mathrm{H}_{1}$ :

$$
\sigma_{\mathrm{F}}=\frac{\mathrm{H}_{1}}{e} \quad \mathrm{~F}=-a\left(\lambda+\sin \varphi_{1}\right) \mathrm{H}_{1} ;
$$

Deuxième cas. - Effort radial $\mathrm{H}_{2}$ :

$$
\sigma_{\mathrm{F}}=\frac{\mathrm{H}_{2}}{e} \quad \mathrm{~F}=-a\left(\lambda+\sin \varphi_{2}\right) \mathrm{H}_{2} ;
$$

Troisième cas. - Efforts axiaux équilibrés :

$$
\sigma_{\mathrm{F}}=\frac{\mathrm{W}_{1}}{e} \quad \mathrm{~F}=a\left(\lambda+\sin \varphi_{1}\right) \mathrm{W}_{1} ;
$$

Quatrieme cas. - Pression équilibrée par $\mathrm{W}_{1}$ :

$$
\sigma_{\mathrm{F}}=\frac{p a}{2} \quad \mathrm{~F}=\frac{p a^{2}}{2}
$$

Cinquième cas. - Moment $\mathrm{M}_{1}$ :

$$
\sigma_{\mathrm{F}}=\frac{6 \mathrm{M}_{1}}{e^{2}} \quad \mathrm{~F}=\mathrm{M}_{1} ;
$$

Sixième cas. - Moment $\mathrm{M}_{2}$ :

$$
\sigma_{\mathrm{F}}=\frac{6 \mathrm{M}_{2}}{e^{2}} \quad \mathrm{~F}=\mathrm{M}_{2} \text {. }
$$

\section{V.8. Les coefficients d'influence s'expriment alors par:}

$\gamma_{\mathrm{N}}=\frac{\sigma_{\mathrm{N}}}{\sigma_{\mathrm{F}}} \quad \gamma_{n}=\frac{\sigma_{n}}{\sigma_{\mathrm{F}}} \quad \gamma_{Q}=\frac{\sigma_{Q}}{\sigma_{\mathrm{F}}} \quad$ pour les efforts

$\gamma_{\mathrm{M}}=\frac{\sigma_{\mathrm{M}}}{\sigma_{\mathrm{F}}} \quad \gamma_{m}=\frac{\sigma_{m}}{\sigma_{\mathrm{F}}}$ pour les moments

$$
\gamma_{y}=\frac{\mathrm{E}_{l}}{\sigma_{\mathrm{F}}} \gamma_{d}=\frac{\mathrm{E} d}{a \sigma_{\mathrm{F}}} \gamma_{f}=\frac{\mathrm{E} f}{a \sigma_{\mathrm{F}}}
$$

pour les déformations

Soit en fonction des valeurs trouvées aux paragraphes V-3 à V-6 et en posant $B_{j}=\frac{F}{a e \sigma_{\mathrm{F}}}$ ( $j$ repère du cas de charge) :

$$
\begin{aligned}
& \gamma_{X}=\frac{\mathrm{B}_{j}}{\lambda+\sin \varphi} \\
& \quad\left[\frac{\beta}{\sin \varphi}+\bar{\omega}(2 \lambda+\sin \varphi)-\mathrm{U} \cot \varphi\right] \\
& \gamma_{n}=-\gamma_{N}+\mathrm{B}_{j} \\
& \left\{\mathrm{I}_{\varphi}-\frac{\rho_{1}}{\rho_{1}-\rho_{2}}\left[\mathrm{I}+\rho_{2}\left(\mathrm{C}_{2}-\mathrm{C}_{1}\right)\right]+2 . \overline{1}\right) \\
& \gamma_{Q}=\frac{3}{2} \mathrm{~B}_{j} \frac{\mathrm{U}}{\lambda+\sin \varphi}
\end{aligned}
$$




$$
\begin{aligned}
\gamma_{\mathrm{M}} & =-6 \frac{a}{e} \dot{B}_{j}\left(\mathrm{~V}^{\prime}+\nu \frac{\cos \varphi}{\lambda+\sin \varphi} \mathrm{V}\right) \\
\gamma_{m} & =-6 \frac{a}{e} \mathrm{~B}_{j}\left(\nu \mathrm{V}^{\prime}+\frac{\cos \varphi}{\lambda+\sin \varphi} \mathrm{V}\right) \\
\gamma_{j} & =k \mathrm{~B}_{j} \mathrm{~V} \\
\gamma_{d} & =\frac{\mathrm{E} d}{a \sigma_{\mathrm{F}}}=(\lambda+\sin \varphi)\left(\frac{n}{e}-\nu \frac{\mathrm{N}}{e}\right) \frac{1}{\sigma_{\mathrm{F}}} \\
=(\lambda+\sin \varphi)\left(\frac{\sigma_{n}}{\sigma_{\mathrm{F}}}-\nu-\frac{\sigma_{\mathrm{N}}}{\sigma_{\mathrm{F}}}\right) & (5-33) \\
\gamma_{d} & =(\lambda+\sin \varphi)\left(\gamma_{n}-\nu \gamma_{\mathrm{N}}\right) \\
\gamma_{f} & =\frac{\mathrm{E} f}{a \sigma_{\mathrm{F}}} \\
& =\int_{\varphi_{1}}^{\varphi}\left[\left(\gamma_{\mathrm{N}}-\nu \gamma_{n}\right) \sin \varphi+\frac{\mathrm{E}}{\sigma_{\mathrm{F}}} y \cos \varphi\right] d \varphi \\
\gamma_{f} & =\int_{\varphi_{1}}^{\varphi}\left[\left(\gamma_{\mathrm{N}}-\nu \gamma_{n}\right) \sin \varphi+\gamma_{y} \cos \varphi\right] \mathrm{d} \varphi
\end{aligned}
$$

Valeur du coefficient $\mathbf{B}_{j}$ pour les différents cas de charges :

$$
\begin{array}{lll}
\text { 1. } & \mathrm{H}_{1} & \mathrm{~B}_{1}=-\left(\lambda+\sin \varphi_{1}\right) \\
\text { 2. } & \mathrm{H}_{2} & \mathrm{~B}_{2}=-\left(\lambda+\sin \varphi_{2}\right) \\
\text { 3. } & \mathrm{W}_{1}, \mathrm{~W}_{2} & \mathrm{~B}_{3}=\lambda+\sin \varphi_{1} \\
\text { 4. } & p, \mathrm{~W}_{1} & \mathrm{~B}_{4}=1 \\
\text { 5. } & \mathrm{M}_{1} & \mathrm{~B}_{5}=\frac{e}{6 a} \\
\text { 6. } & \mathrm{M}_{2} & \mathrm{~B}_{6}=\frac{e}{6 a}
\end{array}
$$

\section{V.9. Calculs numériques des coefficients d'in- fluence.}

\section{V.9.1. CALCUL DE J ET I.}

Nous avons dejjà calculé $\mathrm{J}$ au paragraphe IV-2, formule (4-28).

En appliquant la méthode de Simpson on obtient :

$$
J=\sum_{k=1}^{n} \frac{1+\mu_{2}\left(\lambda \varphi_{k}-\cos \varphi_{k}\right)}{\lambda+\sin \varphi_{k}} \mathbf{Y}_{k i} \Delta_{i k}
$$

où les $\Delta_{k}$ sont ceux utilisés dans le système d'écquations (4-23), (4-24).
On obtient de façon analogue à partir de (5-8) :

$$
I=\sum_{k=1}^{n} \frac{1+0_{2}\left(\lambda \varphi_{k}-\cos \varphi_{k}\right)}{\lambda+\sin \varphi_{k}} Z_{k} \Delta_{k}
$$

\section{V.9.2 Calcul de J et I.}

On a d'après (5-4):

$$
\mathrm{J}_{\varphi}=\int_{\varphi_{1}}^{\varphi} \frac{\mathrm{V}(\alpha)}{\lambda+\sin \varphi} d \alpha
$$

Il s'agit de déterminer la valeur de $\mathrm{J}_{\varphi}$ en chacun des points $1,2,3 \ldots n-1, n$.

Soit $J_{i}$ la valeur numérique de $J_{\varphi}$ au point $\varphi=\varphi_{i}$.

On a évidemment :

$$
\mathrm{J}_{1}=0
$$

Pour calculer $\mathrm{J}_{2}$ avec la méthode de Simpson, nous admettrons que le point représentant la fonction

$$
\frac{V\left(\varphi_{2}\right)}{\lambda+\sin \varphi_{2}}
$$

est situé sur la parabole d'axe $0 \zeta$ passant par les points

$$
\frac{V\left(\varphi_{1}\right)}{\lambda+\sin \varphi_{1}} \text { et } \frac{V\left(\varphi_{3}\right)}{\lambda+\sin \varphi_{3}} \text { (voir fig. 7). }
$$

On a alors :

$$
\begin{aligned}
& J_{2}=\frac{\Delta \varphi}{12} \\
& \times\left[5 \frac{V\left(\varphi_{1}\right)}{\lambda+\sin \varphi_{1}}+8 \frac{V\left(\varphi_{2}\right)}{\lambda+\sin \varphi_{2}}-\frac{V\left(\varphi_{3}\right)}{\lambda+\sin \varphi_{3}}\right]
\end{aligned}
$$

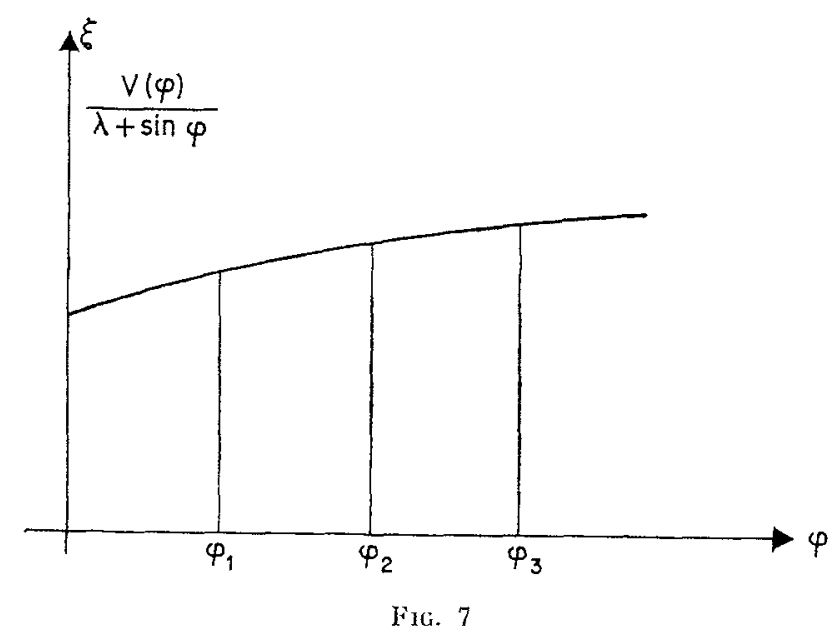


Pour les valeurs suivantes $J_{i}$ est donné par la formule de récurrence :

$$
\begin{aligned}
\mathrm{J}_{i} & =\mathrm{J}_{i-2} \\
+ & \frac{\Delta \varphi}{3}\left[\frac{\mathrm{V}_{i-2}}{\lambda+\sin \varphi_{i-2}}+4 \frac{\mathrm{V}_{i-1}}{\lambda+\sin \varphi_{i-1}}+\frac{\mathrm{V}_{i}}{\lambda+\sin \varphi_{i}}\right]
\end{aligned}
$$

On obtient de même pour $\mathrm{I}_{\varphi}$ :

$I_{1}=0$

$\mathrm{I}_{2}=\frac{\Delta \varphi}{12}\left[5 \frac{Z_{1}}{\lambda+\sin \varphi_{1}}+8 \frac{Z_{2}}{\lambda+\sin \varphi_{2}}-\frac{Z_{3}}{\lambda+\sin \varphi_{3}}\right]$

$\mathrm{I}_{i}=I_{i-2}$

$+\frac{\Delta \varphi}{3}\left[\frac{Z_{i-2}}{\lambda+\sin \varphi_{i-2}}+4 \frac{Z_{i-1}}{\lambda+\sin \varphi_{i-1}}+\frac{Z_{i}}{\lambda+\sin \varphi_{i}}\right]$
V.9.3. Calcul numérioue de la flèche axiale.

Si nous posons

$$
\gamma_{f^{\prime}}=\left(\gamma_{\mathrm{N}}-\gamma \gamma_{n}\right) \sin \varphi+\gamma_{y} \cos \varphi
$$

nous aurons :

$\gamma_{f 1}=0$

$\gamma_{f 2}=\frac{\Delta \omega}{12}\left[5 \gamma_{f^{\prime} 1}+8 \gamma_{f^{\prime} 2}-\gamma_{f^{\prime} 3}\right]$

et :

$\gamma_{f i}=\gamma_{f i-2}+\frac{\Delta Q}{3}\left[\gamma_{f^{\prime} i-2}+4 \gamma_{f^{\prime} i-1}+\gamma_{f^{\prime} i}\right]$

\section{VI. - APPLICATION}

Nous étudierons un élément de soufflet, défini par la figure 8 , et pour lequel

$$
\begin{aligned}
\lambda=\frac{b}{a} & =10 \\
\frac{a}{h} & =20
\end{aligned}
$$

Il suffit de considérer les quarts de tore $O A$ $[0,(\pi / 2)]$ et $O B[\pi,(3 \pi / 2)]$ soumis aux efforts $W$, $H$ et au moment $M$ en $O$. L'effort $W$ est équilibré en $A$ par l'effort axial $W \lambda /(\lambda+1)$ et en $B$ par l'effort $W \lambda /(\lambda-1)$; les rotations en $A$ et $B$ sont nulles.

Un calcul numérique avec 7 points linéairement répartis sur chaque quart de tore donne les coefficients d'influence suivants en $\mathrm{O}$ :

$$
\text { Elément AO : pour } \varphi=0
$$

sous l'effet de l'effort radial $\mathrm{H}$

$$
\begin{array}{ll}
\text { rotation } & \mathrm{V}_{0}{ }^{\mathrm{H}}=-\cdots 405,07687 \\
\text { dilatation radiale } & d_{0}{ }^{\mathrm{H}}=-162,91706
\end{array}
$$

sous l'effet de W équilibré

$$
\begin{array}{ll}
\text { rotation } & \mathrm{V}_{0}{ }^{w}=1705,7363 \\
\text { dilatation radiale } & d_{0}{ }^{w}=305,43103
\end{array}
$$

sous l'effet du moment $M$

$$
\begin{aligned}
& \text { rotation } \\
& \mathrm{V}_{0}{ }^{\mathrm{MI}}=31,78201
\end{aligned}
$$

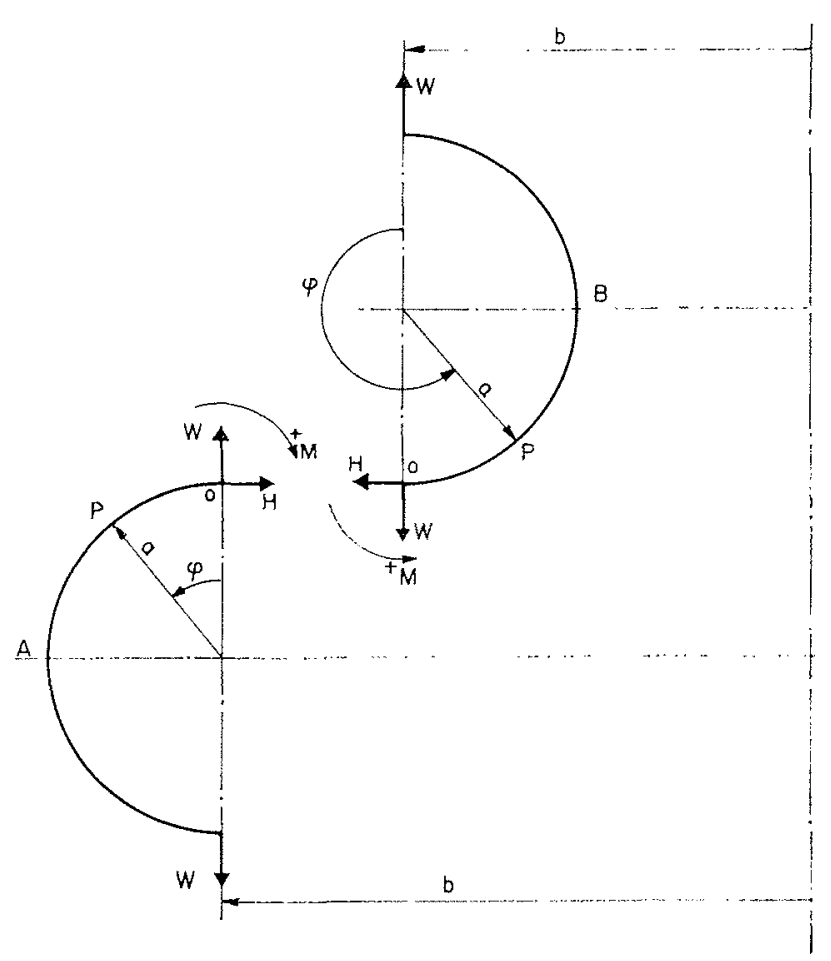

FIG. 8 
Elément $\mathrm{OB}$ : pour $\bullet=\pi$

sous l'effet de $\mathrm{H}$

$$
\begin{array}{lll}
\text { rotation } & V_{\pi}^{H}=- & 400,06262 \\
\text { dilatation radiale } & d_{\pi}^{\mathrm{H}}= & 152,16563
\end{array}
$$

sous l'effet de W équilibré

$$
\begin{array}{ll}
\text { rotation } & \mathrm{V}_{\pi}{ }^{\mathrm{W}}=\mathbf{1 6 6 7 , 1 5 3 5} \\
\text { dilatation radiale } & d_{\pi}{ }^{\mathrm{W}}=--305,76133
\end{array}
$$

sous l'effet de $\mathrm{M}$

$$
\begin{array}{ll}
\text { rotation } & \mathrm{V}_{\pi}{ }^{\mathrm{M}}=-31,57294 \\
\text { dilatation radiale } & d_{\pi^{M}}=
\end{array}
$$

L'égalité des rotations et des dilatations radiales en $\mathrm{O}$ donne le système de deux équations :

$-5,01425 \frac{\mathrm{H}}{h}+38,5828 \frac{\mathrm{W}}{h}+63,35495 \frac{6 \mathrm{M}}{h^{2}}=0$

$-315,08269 \frac{\mathrm{H}}{h}+611,19236 \frac{\mathrm{W}}{h}+0,041785 \cdot \frac{6 \mathrm{M}}{h^{2}}=0$

soit :

$$
\frac{\left(\frac{\mathrm{H}}{h}\right)_{0}=1,93973 \frac{\mathrm{W}}{h}}{\left(\frac{6 \mathrm{M}}{h^{2}}\right)_{0}=-0,455474 \frac{\mathrm{W}}{h}}
$$

Connaissant les efforts à la liaison, on détermine ensuite les efforts et déformations aux différents points $P_{i}$ de la méridienne. Ces valeurs sont représentées sur les graphiques 9 à 15 en fonction de $\sigma_{W}=(W / h)$.

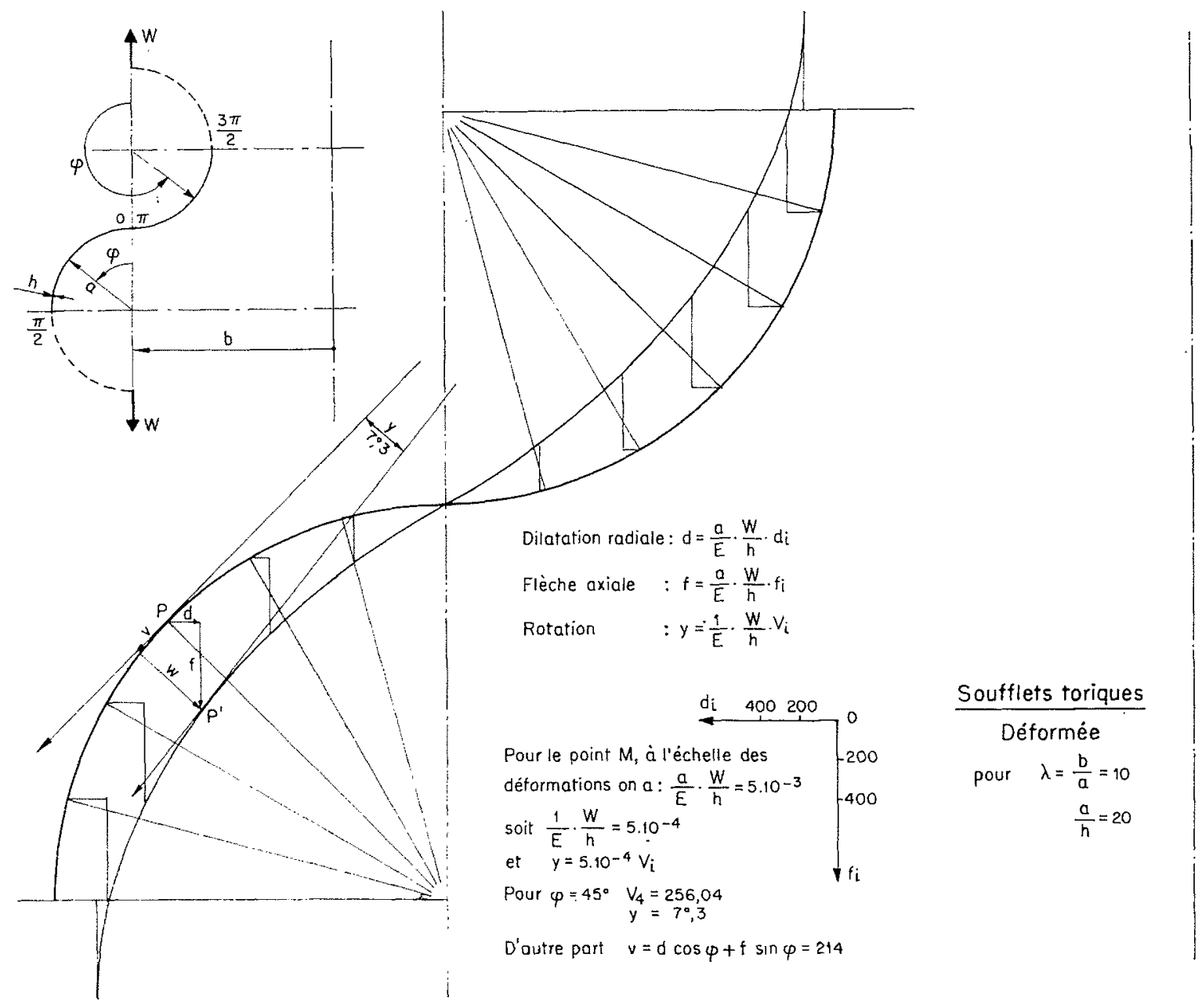

Fig. 0 


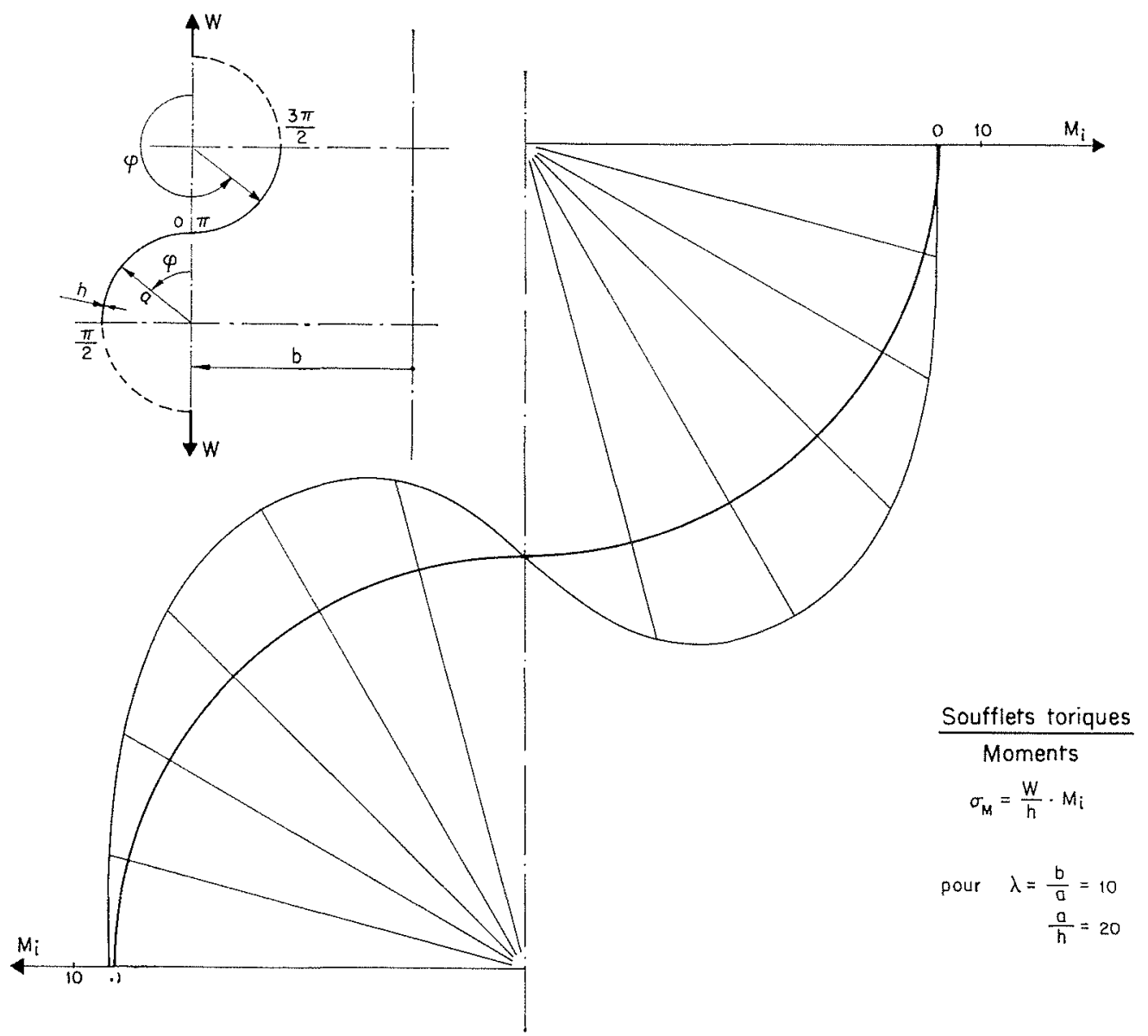

FIG. 10
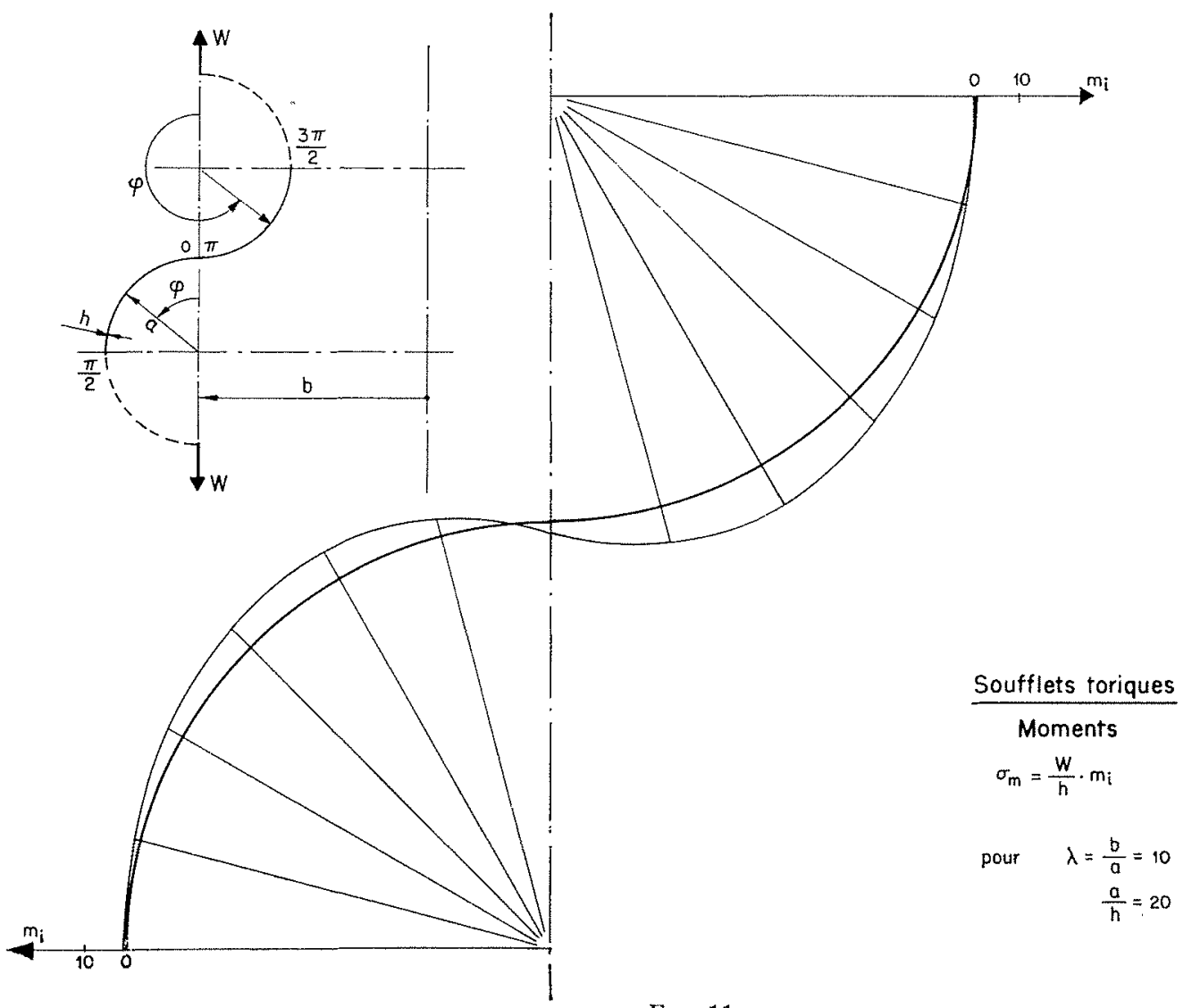

Fig. 11 


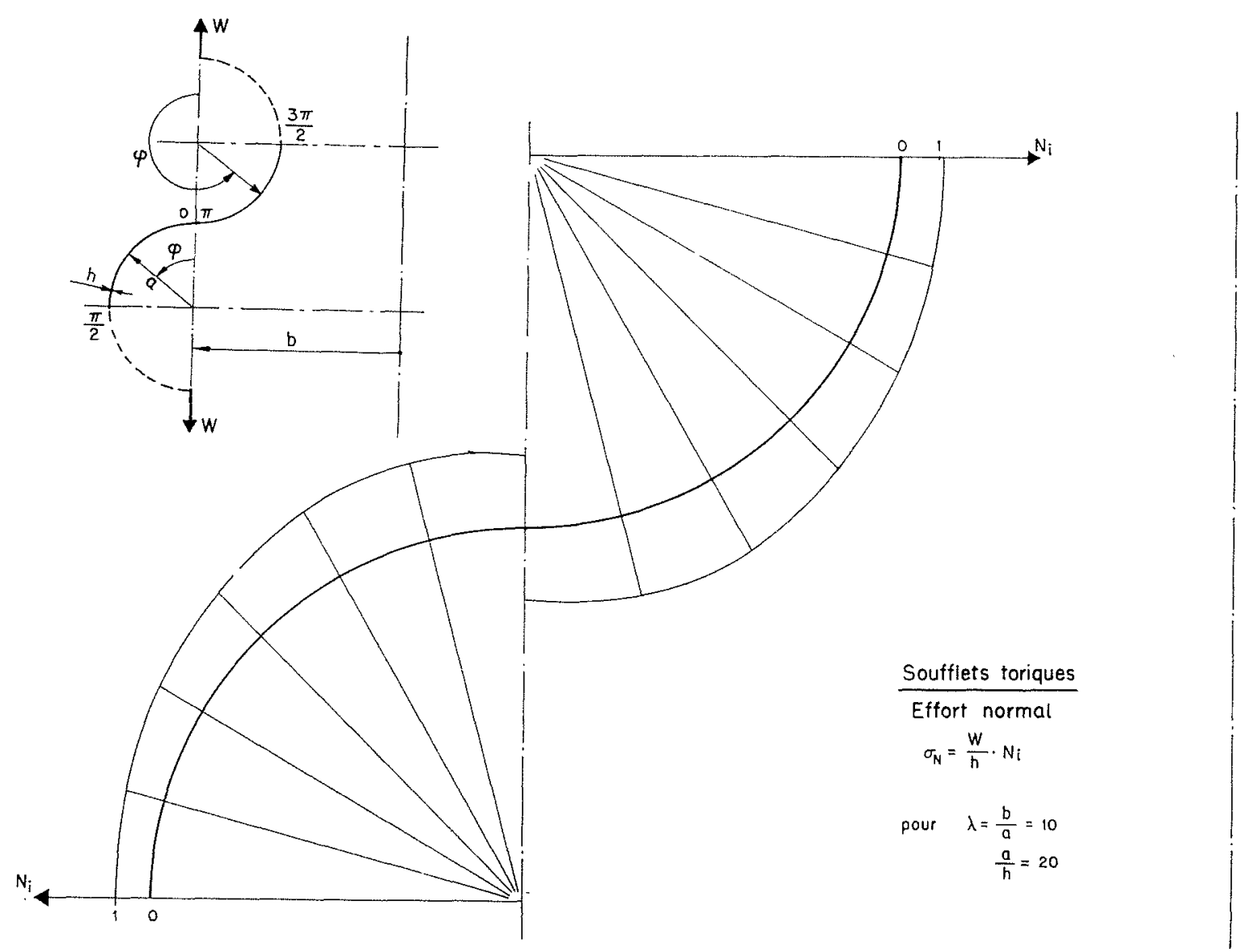

FIG. 12

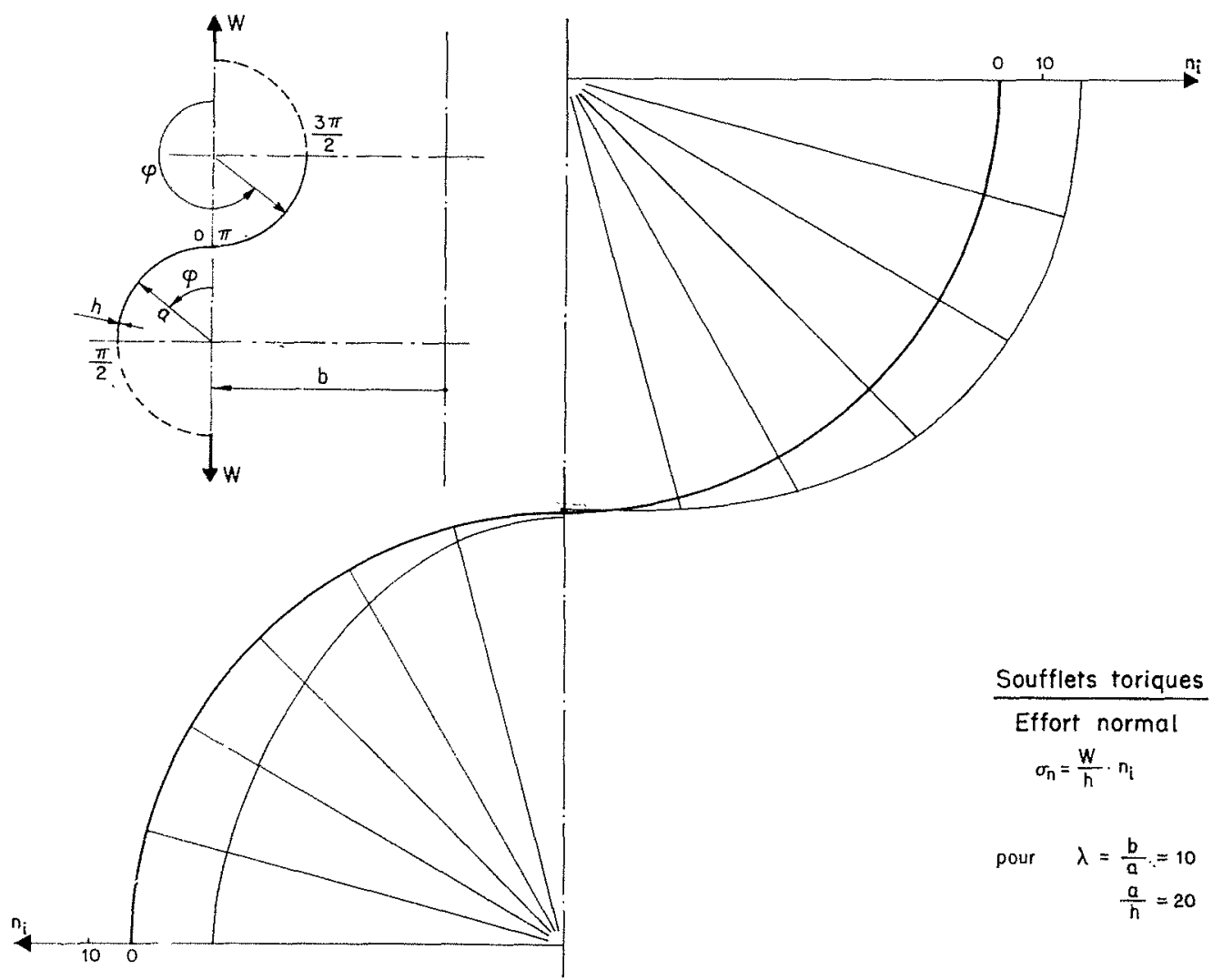

FIG. 13 

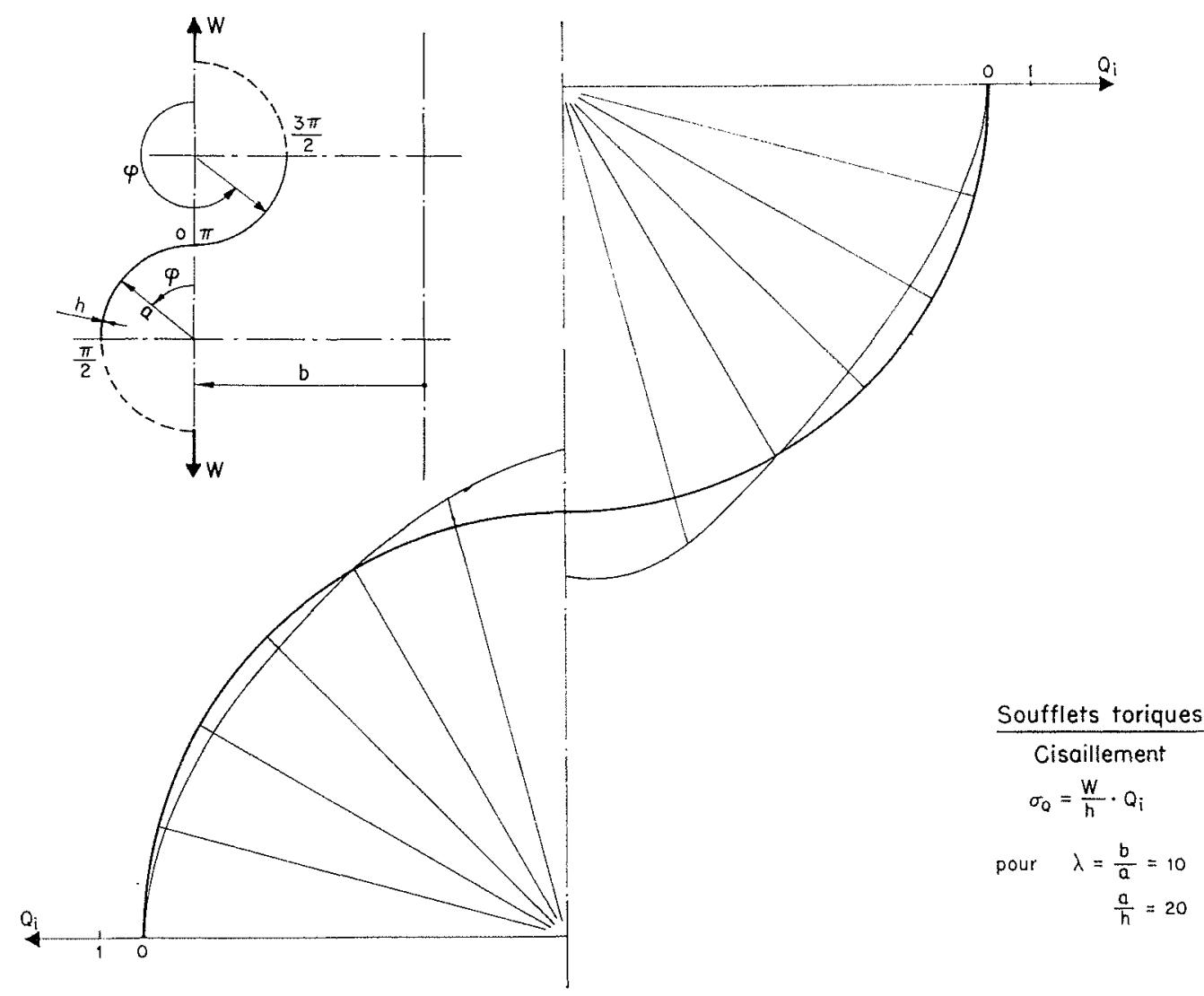

FIG. 14

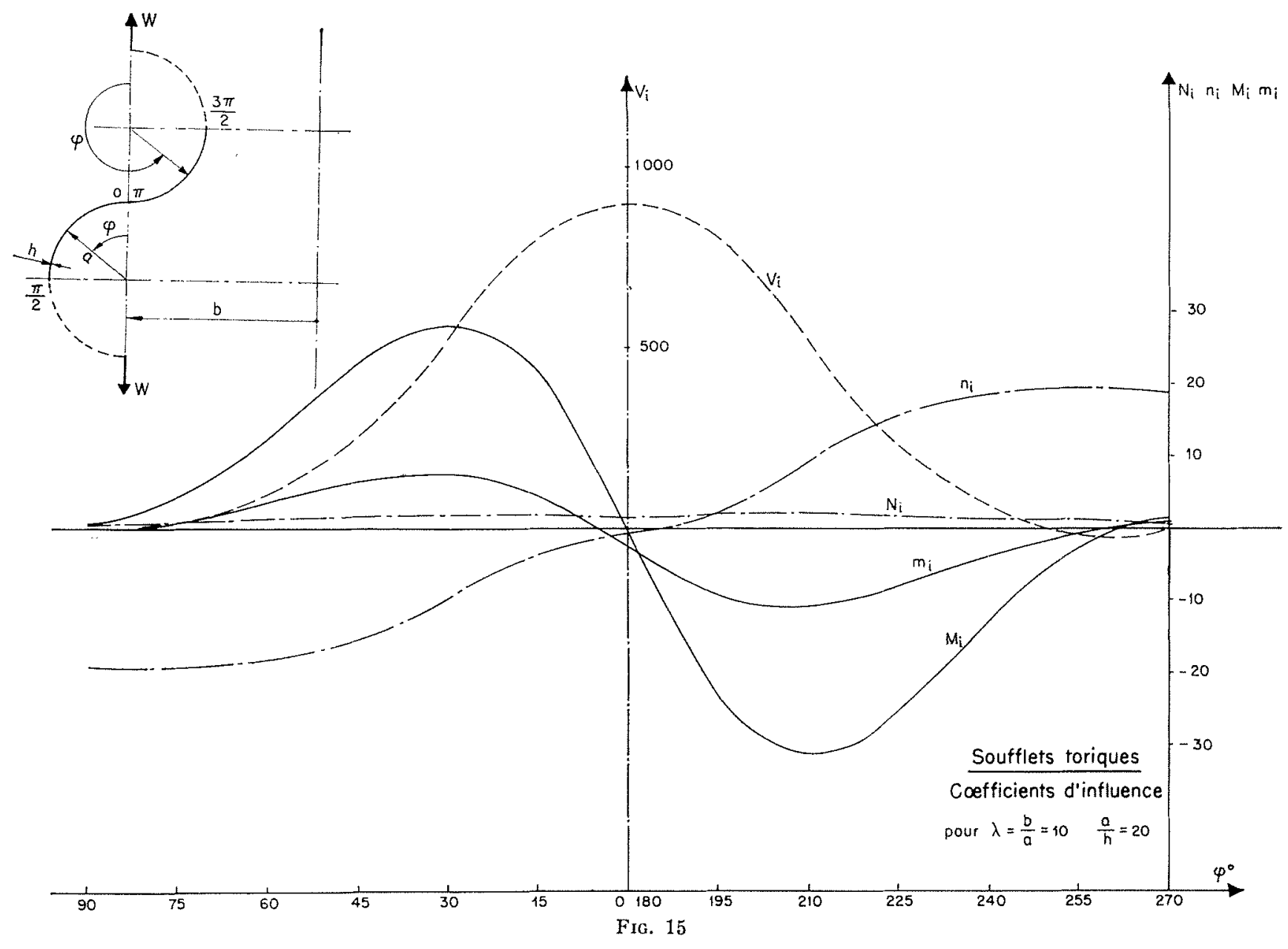




\section{VII. - CONCLUSION}

Dans le cadre des hypothèses admises pour la théorie de la flexion des coques minces, la móthode exposée permet de calculer les coques toriques quels que soient les paramètres qui les dofinissent :

- Rapport $\lambda$ entre le rayon d'enroulement ef le rayon du méridien;

- Rapport $a / h$ entre le rayon du méridien et l'épaisseur de la coque;

- Caractéristiques du matériau;

- Angles au centre $\varphi_{1}$ et $\varphi_{2}$ définissant l'arc de méridien.
Nous n'avons pas pu comparer complètement nos résultats avec ceux de Clark. Dans son étude, Clark ne donne que la position et la valeur approximatives des déformations. Les données sont pratiquement confirmées par nos résultats.

Pour les cas de charge particuliers étudiés par Wissler et repris par Clark, nos résultats sont en bon accord avec ceux de ces deux auteurs.

La même méthode peut s'appliquer à des coques toriques d'épaisseur variable; voir, par exemple, l'étude des auteur's citée dans la bibliographie sous le numéro [2].

\section{BIBLIOGRAPHIE}

11] S. Casacci. - Etude de la flexion des coques de révolution chargées axisymétriquement. Travaux, novembre-décembre 1959 .

[2] S. Casachi et G. Picollier. - Etude de la flexion des coques coniques d'épaisseur variable soumises à des efforts centrifuges. La Houille Blanche, $\mathrm{n}^{*} \mathrm{1}$, janvier-février 1961, pp. 46-58.

[3] V.S. Cernina, - Etat de contrainte d'une enveloppe en forme de tore d'épaisseur non négligeable. Izvestija Akademii Nauk SSSR, Mekhanika i Masinostroenie, $\mathrm{n}^{\circ} 3,1959$, pp. 96-104.

[4] R. A. Clark. - On the theory of thin elastic toroidal shells. Journal of Mathematics and Physics, vol. 29,1950 , pp. 146-177.

[5] R. A. Clark and E. Reissner. - A problem of finite bending of toroidal shelis. Quaterly of Applied Mathematics, vol. X, 1952, pp. 321-334.

6] R.A. Clark, Gilroy and E. Reissner. - Stresses and deformations of toroidal shells of elliptical cross section. Journal of Applied Mechanics, vol. 19,1952, p. 37

[7] G. D. Gallety. - Torispherical shells, - A caution to designers. A.S.M.E., Paper $\mathbf{n}^{\circ}$ 58, PET. 3.
On particular integrals for toroidal shells subjected to uniform internal pressure. Journat of Applied Mechanics, vol. 25, $\mathrm{n}^{\circ} 3$, September 1958 , p. 412 .

$A$ comparison of methods for analysing bending effects in toroidal shells. Journal of Applied Mechanics, vol. 25, no 3, September 1958, p. 413.

[8] P. V. Marchal and C.E. Turner. - Elastic solution in the limit analysis of shells of revolution with special reference to expansion bellows. Journal of Mechanical Engineering Science, vol. 3, $\mathrm{n}^{\circ}$ 3, 1961, pp. $252-257$.

[9] R.T. Shields and D. C. Brucien. - Limit strength of thin walled pressure vessels with an ASMF standard torispherical head. Proceeding of the third U.S. National Congress of applied Mechanics, 1958, pp. 665-672.

[10] K. Stange. - Der Spannungszustand einer Kreisringschale. Ingenieur Archiv, 2, 1932, pp. 47-91.

[11] L. N. TAO. - On toroidal shells. Journal of Mathematics and Physics, vol. XXXVIII, n: 2, July 1959 , pp. 130-134.

[12] H. Wissler. - Festigkeitsberechnung von Ringflachenschalen. Thèse de doctorat, Zurich, 1916. 